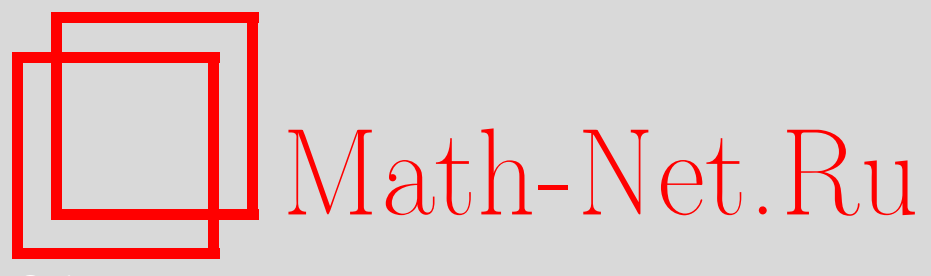

А. Ю. Морозов, М. Н. Сербин, Нелинейная алгебра и рекурсия Боголюбова, ТМФ, 2008, том 154, номер 2, 316343

DOI: https://doi.org/10.4213/tmf6172

Использование Общероссийского математического портала Math-Net.Ru подразумевает, что вы прочитали и согласны с пользовательским соглашением http: //www . mathnet.ru/rus/agreement

Параметры загрузки:

IP : 54.174 .149 .18

26 апреля 2023 г., $12: 22: 52$

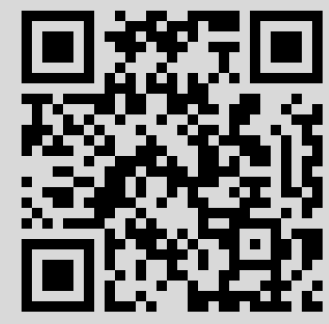




\title{
НЕЛИНЕЙНАЯ АЛГЕБРА И РЕКУРСИЯ БОГОЛЮБОВА
}

\begin{abstract}
Приведены многочисленные примеры применения формулы лесов Боголюбова к итеративному решению различных нелинейных уравнений. При этом одна и та же формула описывает чрезвычайно широкий класс объектов, начиная с обыкновенного квадратного уравнения и заканчивая перенормировками в квантовой теории поля.
\end{abstract}

Ключевые слова: квантовая теория поля, перенормировки, нелинейная алгебра.

\section{1. ВВЕДЕНИЕ}

Формула лесов Боголюбова [1]-[5] является одним из краеугольных камней квантовой теории поля и используется при перенормировках. В работах [6] и, главным образом, [7] было показано, что формула лесов является чисто алгебраической конструкцией, разработанной для итеративного решения широкого класса нелинейных уравнений. При этом замечательно, что одна и та же формула описывает необычайно широкий круг задач, начиная с решения обычного квадратного уравнения и заканчивая лэмбовским сдвигом и перенормировкой Стандартной модели. Общее рассмотрение в работе [7] является слишком абстрактным и не содержит примеров. Несколько примеров можно встретить в работах [8]-[14], но все они относятся к квантовой теории поля и поэтому скрывают простую алгебраическую и тензорную структуры формулы лесов. Учебный обзор в работе [15] рассматривает простые примеры, однако там не уделено достаточно внимания алгебре и формуле лесов. Цель этой статьи - дать необходимые примеры применения формализма работы [7], что является естественным дополнением к общему рассмотрению нелинейной алгебры, которое содержится в работе [16].

${ }^{*}$ Институт теоретической и экспериментальной физики, Москва, Россия. E-mail: morozov@itep.ru

${ }^{\dagger}$ Museo Storico della Fisica e Centro Studi e Ricerche "Enrico Fermi", Rome, Italy; Mocковский физико-технический институт; Институт теоретической физики им. Л. Д. Ландау РАН, Москва, Россия; Институт теоретической физики им. Н. Н. Боголюбова, Киев, Украина. E-mail: maksym.serbyn@itep.ru 
1.1. Постановка проблемы. Формула лесов дает итеративное решение следующей задачи.

- Пусть $F(T)$ - заданная функция переменных $T$, называемых временами или константами связи. В применениях к квантовой теории поля $F(T)$ обычно является эффективным действием (логарифмом статсуммы) физической теории, рассматриваемым как функция ее голых констант связи, но эта интерпретация несущественна для формулы лесов как таковой.

- Пусть $\mathcal{P}_{-}$- линейный проектор на линейное подпространство нежелателъных (например, сингулярных) функций. В контексте теории перенормировок функция $F(T)$ зависит также от обрезания $\Lambda$, и нежелательными являются функции, бесконечные в пределе $\Lambda \rightarrow \infty$. Однако опять-таки конкретная интерпретация проектора $\mathcal{P}_{-}$несущественна для формулы лесов.

- При данных $F(T)$ и $\mathcal{P}_{-}$мы уже можем сформулировать проблему. Можно ли сделать замену аргументов $T$ в $F(T)$, не меняя вида этой функции, чтобы перевести ее в пространство желательных (несингулярных) функций? Другими словами, проблема состоит в нахождении замены переменных $T \rightarrow \widetilde{T}=T+Q(T)$ такой, что

$$
\mathcal{P}_{-}\{F(T+Q(T))\}=0 \text {. }
$$

Чтобы сделать решение этой задачи однозначным, следует наложить дополнительное условие, состоящее в том, что контрчлены $Q(T)$ являются чисто сингулярными, т.е. полностью лежат в пространстве нежелательных функций:

$$
\mathcal{P}_{+}\{Q(T)\}=0
$$

где $\mathcal{P}_{+}=I-\mathcal{P}_{-}-$проектор на пространство несингулярных функций ${ }^{1)}$.

\section{2. Формула лесов: решение проблемы на языке фейнмановских диа-} грамм. Формула лесов дает явное решение задачи (1), (2) на языке теории графов. Главное наблюдение, сделанное в работе [7], состоит в том, что любой ряд из неотрицательных степеней констант связи $T$ может быть представлен как сумма фейнмановских диаграмм, в которых $T$ стоят в вершинах, что дает наиболее подходящий язык для изучения тензорной алгебры [16]; это будет ясно из дальнейших примеров. Таким образом, как $F(T)$, так и контрчлен $Q(T)$ могут быть разложены в суммы по диаграммам вида

$$
F(T)=\sum_{\Gamma} \widehat{F}(\Gamma) Z(\Gamma \mid T), \quad Q(T)=\sum_{\Gamma} \widehat{Q}(\Gamma) Z(\Gamma \mid T),
$$

в которых базисные функции $Z(\Gamma \mid T)$ описывают топологию диаграммы $\Gamma: Z(\Gamma \mid T)-$ это произведение констант связи $T$, стоящих в вершинах с индексами (если таковые имеются), свернутое вдоль ребер. Мы предполагаем что проекторы $\mathcal{P}_{ \pm}$действуют

\footnotetext{
1) В действительности это условие является несколько более сложным: $\mathcal{P}+\{\widehat{F}(\Gamma / \Gamma) \widehat{Q}(\Gamma)\}=0$ (см. п.п. I.1.4.1 и I.1.4.2 настоящей работы).
} 
на коэффициенты $\widehat{F}(\Gamma)$ и $\widehat{Q}(\Gamma)$, а не на $Z(\Gamma \mid T)$, и это в самом деле имеет место в большинстве случаев.

Теперь итеративное решение задачи (1), (2) дается формулой лесов

$$
\widehat{F}(\Gamma / \Gamma) \widehat{Q}(\Gamma)=-\mathcal{P}_{-}\left\{\widehat{F}(\Gamma)+\sum_{\left\{\gamma_{1} \cup \ldots \cup \gamma_{k}\right\}} \widehat{F}\left(\Gamma /\left(\gamma_{1} \ldots \gamma_{k}\right)\right) \widehat{Q}\left(\gamma_{1}\right) \ldots \widehat{Q}\left(\gamma_{k}\right)\right\}
$$

где $\left\{\gamma_{1} \cup \cdots \cup \gamma_{k}\right\}$ - это все возможные прямоугольные подграфы графа $\Gamma, \gamma_{i} \in \mathcal{B} \Gamma$, т.е. $\gamma_{i}$ суть части $\Gamma$, лежащие в совокупности непересекающихся прямоугольников, а $\Gamma /\left(\gamma_{1} \ldots \gamma_{k}\right)$ получается стягиванием всех прямоугольников в точки. Множитель $\Gamma / \Gamma$ - это просто одна вершина с валентностью, равной числу внешних ног графа $\Gamma$, и $\widehat{Q}(\Gamma) \neq 0$ задается уравнением $(4)$, только если $\widehat{F}(\Gamma / \Gamma) \neq 0$. Если $\widehat{Q}(\Gamma) \neq 0$ для какого-то графа $\Gamma$, то в $F(T)$ должна присутствовать соответствующая константа связи $\widehat{F}(\Gamma / \Gamma) \neq 0$. Мы назовем это правило вершинным критерием. Оно будет играть важную роль в дальнейших рассуждениях.

Обсуждение алгебраической теории, лежащей в основе уравнения (4), а также связи этого уравнения с группой диффеоморфизмов пространства модулей теорий и с дуальной алгеброй Хопфа-Конна-Краймера на графах можно найти в работах [6], [7]. Мы же в дальнейшем будем рассматривать многочисленные примеры применения уравнения (4).

1.3. Формула лесов второго уровня. Помимо формулы лесов первого уровня (4) существует формула Боголюбова следующего уровня [1]-[3], включающая в себя сумму по вложенным прямоугольным подграфам (см. уравнение (9.7) в работе [7]). Формула лесов второго уровня напрямую дает ответ для перенормированного эффективного действия $F(\widetilde{T})=F(T+Q(T))=\sum_{\Gamma} \widehat{F}_{\mathrm{R}}(\Gamma) Z(\Gamma \mid T)$ :

$$
\widehat{F}_{\mathrm{R}}(\Gamma)=\sum_{\mathcal{F}_{\Gamma}} \prod_{\mathcal{T} \in \mathcal{F}_{\Gamma}}\left(\prod_{\substack{\text { vertices } \\ \text { of } \mathcal{T}}} \frac{1}{\widehat{F}\left(\hat{\gamma}_{k} / \hat{\gamma}_{k}\right)}\left(-\mathcal{P}_{-}\right) \widehat{F}\left(\hat{\gamma}_{k} /\left(\hat{\gamma}_{k+1}^{1} \ldots \hat{\gamma}_{k+1}^{s(k)}\right)\right)\right)
$$

Здесь сумма идет по всевозможным лесам $\mathcal{F}_{\Gamma}$ (лесом называется объединение деревьев для всех связных компонент графа Г). Для $\Gamma_{1}$ (связной компоненты графа Г) дерево $\mathcal{T}$ строится следующим образом.

1. Выбирается определенный набор вложенных прямоугольных подграфов $\left\{\gamma_{n}\right\}$ :

$$
\gamma_{0}=\Gamma_{1}, \quad \gamma_{1} \in \mathcal{B} \Gamma, \quad \gamma_{2} \in \mathcal{B} \gamma_{1} \subset \mathcal{B} \Gamma, \quad \ldots, \quad \gamma_{n} \in \mathcal{B} \gamma_{n-1} \subset \cdots \subset \mathcal{B} \gamma_{1} \subset \mathcal{B} \Gamma_{1},
$$

где граф $\gamma_{k}$ не обязательно должен быть связным и может состоять из $s(k)$ связных компонент $\gamma_{k}^{1}, \ldots, \gamma_{k}^{s(k)}$. Однако для каждой связной компоненты $\gamma_{k}^{i}$ должна существовать связная компонента $\gamma_{k-1}^{j(i)}$ такая, что $\gamma_{k}^{i} \in \mathcal{B} \gamma_{k-1}^{j(i)}$ (условие (6) следует понимать именно таким образом).

2. Используя этот набор вложенных прямоугольных подграфов или, что то же самое, набор непересекающихся прямоугольников, мы строим декорированное корневое дерево $\mathcal{T}$. Нижней стороне каждого из прямоугольников ставится в соответствие 
вершина дерева. Две вершины связаны друг с другом, если один из прямоугольников лежит непосредственно в другом (т.е. между ними нет других прямоугольников). Корень дерева расположен в вершине, связанной с $\gamma_{0}=\Gamma$. На языке графов это означает, что каждая вершина дерева связана с определенным прямоугольным подграфом $\gamma_{k}^{i}$ и существует только одно ребро, направленное вниз (т.е. к корню) и связанное с подграфом, непосредственно в котором лежит данный граф, и связывающее $\gamma_{k}^{i}$ с $\gamma_{k-1}^{j(i)}$. При этом имеется произвольное число ребер, направленных вверх и связывающих $\gamma_{k}$ с подмножеством $\gamma_{k+1}^{1}, \ldots, \gamma_{k+1}^{s(k, i)} \subset \gamma_{k+1}$.

Произведение вдоль дерева

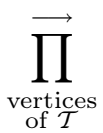

ставит в соответствие каждой вершине дерева $\gamma_{k}^{i}$ оператор $\left(\widehat{F}\left(\left[\gamma_{k}^{i} / \gamma_{k}^{i}\right]\right)\right)^{-1}\left(-\mathcal{P}_{-}\right) \times$ $\widehat{F}\left(\gamma_{k}^{i} /\left(\gamma_{k+1}^{1} \ldots \gamma_{k+1}^{j(i)}\right)\right)$, причем проектор $\mathcal{P}_{-}$действует вверх вдоль ветвей дерева. Корневая вершина $\gamma_{0}$ (т.е. связная компонента графа Г) дает множитель $\widehat{F}\left(\gamma_{0} /\left(\gamma_{1}^{1} \ldots \gamma_{1}^{s(0)}\right)\right)$. Стрелка над знаком произведения означает, что произведение упорядочено вдоль ветвей дерева.

Задача $(1),(2)$ подразумевает что функция $F(T)$ и проектор $\mathcal{P}_{-}$уже заданы. Однако надо еще выбрать диаграммную технику. Для заданных $F(T)$ и $\mathcal{P}$ - это можно сделать различными способами. Также стоит отметить, что итеративность формулы лесов вовсе не подразумевает наличие малого параметра разложения; более того, в контексте теории перенормировок существенный параметр разложения является большим, а не малым.

\section{І. АЛГЕБРАИЧЕСКИЕ ПРИМЕРЫ}

\section{I.1. КВАДРАТНОЕ УРАВНЕНИЕ. ДВУХВАЛЕНТНАЯ ВЕРШИНА И ДРЕВЕСНЫЕ ДИАГРАММЫ}

Исходным примером послужит задача

$$
\begin{gathered}
F(T)=T+\epsilon T^{2}, \\
\mathcal{P}_{-}\{f(\epsilon)\}=f(\epsilon)-f(0),
\end{gathered}
$$

т.е. нежелательными являются функции, зависящие от $\epsilon$. Мы хотим исключить зависимость от $\epsilon$, совершая (зависящие от $\epsilon$ ) сдвиги переменной $T \rightarrow \widetilde{T}+Q_{\epsilon}(T)$ так, чтобы функция $F(\widetilde{T})=\widetilde{T}+\epsilon \widetilde{T}^{2}$ больше не зависела от $\epsilon$. Налагая дополнительное условие (2), мы сведем задачу к уравнению

$$
\widetilde{T}+\epsilon \widetilde{T}^{2}=T
$$

таким образом,

$$
\begin{aligned}
\widetilde{T} & =\frac{\sqrt{1+4 \epsilon T}-1}{2 \epsilon}=T+\sum_{k=1} \frac{(-1)^{k} 2^{k}(2 k-1) ! !}{(k+1) !} \epsilon^{k} T^{k+1}= \\
& =T-\epsilon T^{2}+2 \epsilon^{2} T^{3}-5 \epsilon^{3} T^{4}+14 \epsilon^{4} T^{5}-\cdots .
\end{aligned}
$$


Теперь мы собираемся воспроизвести этот ряд, используя формулу лесов (4). Для этого нам сначала следует выбрать определенную диаграммную технику. Сначала выберем одну двухвалентную вершину $T=T_{i j}$, где индексы $i, j$ принимают всего одно значение, $i=j=1$, и в дальнейшем будут опускаться. Единственно возможные в данном случае связные фейнмановские диаграммы - это цепочечные диаграммы и окружности, соответствующие $T^{n}$, где $n$ - число вершин. В $F(T)$ дают вклад только две диаграммы: с $n=1$ и $n=2$.

I.1.1. Цепочечные диаграммы. Сначала рассмотрим цепочечные диаграм$\mathrm{MbI}^{2)}$

$$
\widehat{F}(\bullet)=1, \quad \widehat{F}(\bullet \bullet)=\epsilon .
$$

Соответственно $F(\Gamma / \Gamma) \neq 0$ только для цепочечных диаграмм $Г$. Согласно вершинному критерию это означает что ненулевой вклад в $Q$ дают только цепочечные диаграммы. Для них из формулы лесов (4) получаем

$$
\begin{aligned}
Q[1] & \equiv Q(\bullet-)=-\mathcal{P}_{-}\{F(\bullet-)\}=0, \\
Q[2] & \equiv Q(\bullet \bullet)=-\mathcal{P}_{-}\{F(\bullet \bullet)\}=-\epsilon, \\
Q[3] & \equiv Q(\bullet \bullet \bullet-)=-\mathcal{P}_{-}\{F(\bullet \bullet \bullet-)+2 F(\bullet \bullet) Q(\bullet \bullet-)\}= \\
& =-2 \mathcal{P}_{-}\{F(\bullet \bullet) Q(\bullet \bullet)\}=+2 \epsilon^{2} .
\end{aligned}
$$

В последней формуле $F(\bullet \bullet \bullet)=0$ вследствие нашего выбора $F(T)$, а коэффициент 2 перед следующим слагаемым появляется из-за наличия двух способов выбора

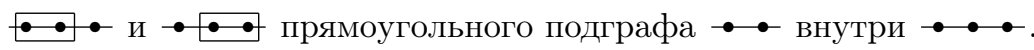

Аналогично, имеются три прямоугольных подграфа $\bullet \bullet \bullet \bullet, \bullet \bullet \bullet и$ $\bullet \bullet$, которые дают вклад в следующее $Q$ :

$$
Q[4] \equiv Q(\bullet \bullet \bullet \bullet)=-\mathcal{P}_{-}\left\{F(\bullet \bullet)\left(2 Q(\bullet \bullet \bullet)+[Q(\bullet \bullet)]^{2}\right)\right\}=-5 \epsilon^{3} .
$$

При этом подграфы $\bullet \bullet \bullet \bullet$ и $\bullet \bullet \bullet-$ не дают вклада, так как после стягивания прямоугольников получаются трехвершинные диаграммы, и этот вклад умножается на значение $F(\bullet \bullet \bullet)$, которое равно нулю в силу выбора $F(T)$. Обозначим $N$-вершинную линейную диаграмму как $[N]$. Тогда

$$
Q[5]=-\mathcal{P}_{-}\{F[2](2 Q[4]+2 Q[2] Q[3])\}=14 \epsilon^{4},
$$

и в общем случае уравнение (4) дает

$$
Q[N]=-\mathcal{P}_{-}\left\{F[2]\left(\delta_{N, 2}+2 Q[N-1]+\sum_{k=2}^{N-2} Q[k] Q[N-k]\right)\right\}, \quad N \geqslant 3 .
$$

\footnotetext{
${ }^{2)}$ В дальнейшем мы будем опускать шляпки над $\widehat{Q}(\Gamma)$ и $\widehat{F}(\Gamma)$, так как в соответствующем контексте их легко отличить от функций $Q(T)$ и $F(T)$ переменной $T$.
} 
Для производящей функции $Q(t)=\sum_{N=2} t^{N} Q[N]$ получаем

$$
Q(t)=-F[2]\left(t^{2}+2 t Q(t)+Q^{2}(t)\right)
$$

где $F[2]=\epsilon$, т.е. мы имеем знакомое нам уравнение $(9)$ :

$$
Q(t)+t+\epsilon(Q(t)+t)^{2}=t, \quad Q(t)+t=\frac{\sqrt{1+4 \epsilon t}-1}{2 \epsilon} .
$$

I.1.2. Введение коэффициентов. В предыдущем примере базисные функции $Z(\Gamma \mid T)$ входили в первоначальную функцию $F(T)$ с коэффициентами 1 и $\epsilon$. Если вместо этого мы напишем

$$
F(T)=a \bullet+\epsilon b \bullet \bullet=a T+\epsilon b T^{2}, \quad \text { т.e. } \quad \widehat{F}(\bullet)=a, \quad \widehat{F}(\bullet \bullet)=\epsilon b,
$$

то коэффициент $а$ появится слева в уравнении (4):

$$
\begin{aligned}
a Q(\bullet-) & =-\mathcal{P}_{-}\{F(\bullet-)\}=0 \\
a Q(\bullet \bullet) & =-\mathcal{P}_{-}\{F(\bullet \bullet)\}=-\epsilon b \\
a Q(\bullet \bullet \bullet) & =-2 \mathcal{P}_{-}\{F(\bullet \bullet) Q(\bullet \bullet)\}=\frac{2 \epsilon^{2} b^{2}}{a},
\end{aligned}
$$

так что

$$
\widetilde{T}=T+Q(\bullet) T+Q(\bullet \bullet) T^{2}+Q(\bullet \bullet \bullet) T^{3}+\cdots=T-\frac{\epsilon b T^{2}}{a}+\frac{2 \epsilon^{2} b^{2} T^{3}}{a^{2}}-\cdots
$$

и

$F(\widetilde{T})=a \widetilde{T}+\epsilon b \widetilde{T}^{2}=a\left(T-\frac{\epsilon b T^{2}}{a}+\frac{2 \epsilon^{2} b^{2} T^{3}}{a^{2}}-\cdots\right)+\epsilon b\left(T-\frac{\epsilon b T^{2}}{a}+\frac{2 \epsilon^{2} b^{2} T^{3}}{a^{2}}-\cdots\right)^{2}=a T$.

I.1.3. Применение формулы лесов второго уровня. Формула лесов второго уровня (5) непосредственно дает перенормированную функцию $F(\widetilde{T})$. Из уравнения (14) нам уже известно, чему она равна, и ответ очень прост:

$$
F(\widetilde{T})=F_{\mathrm{R}}(T)=a T .
$$

Это означает, что в нашем случае формула (5) дает единственное ненулевое значение $F_{\mathrm{R}}(-\bullet)=a$, а для всех остальных графов $F_{\mathrm{R}}(\Gamma)=0$. Первое утверждение с очевидностью следует из самой формулы: в данном случае в правой части (5) присутствует всего одно слагаемое. Для следующих графов Г должно происходить точное сокращение различных вкладов из (5).

Покажем, как это происходит для графа $\bullet \bullet-$, для которого можно построить следующие два дерева: 
Мы не рассматриваем деревья с прямоугольными подграфами, содержащими одну вершину, наподобие $\bullet$, поскольку $\mathcal{P}_{-} F(\bullet)=0$. Мы также не изображаем внешний прямоугольник, который соответствует всему графу. Таким образом,

$$
F_{\mathrm{R}}(\bullet \bullet)=F(\bullet \bullet)+F(\bullet) \frac{1}{F(\bullet)}\left(-\mathcal{P}_{-}\right) F(\bullet \bullet)=F(\bullet \bullet)-\mathcal{P}_{-} F(\bullet \bullet)=0 .
$$

У графа ••-•- имеются следующие наборы вложенных прямоугольных подграфов (показаны только те, которые дают ненулевой вклад в (5)): $\bullet \bullet \bullet$,

$\bullet \bullet \bullet$. Очевидно, что первые два дерева дают такой же вклад, как и два последних, поэтому мы учитываем только первые два графа, умножая результат на 2:

$$
\begin{aligned}
F_{\mathrm{R}}(\bullet \bullet-)=2 & \left\{F(\bullet \bullet) \frac{1}{F(\bullet-)}\left(-\mathcal{P}_{-}\right) F(\bullet \bullet)+\right. \\
& \left.+F(\bullet-) \frac{1}{F(\bullet-)}\left(-\mathcal{P}_{-}\right)\left[F(\bullet \bullet) \frac{1}{F(\bullet-)}\left(-\mathcal{P}_{-}\right) F(\bullet \bullet-)\right]\right\} .
\end{aligned}
$$

Поэтому

$$
F_{\mathrm{R}}(\bullet \bullet-)=-\frac{2}{F(\bullet)}\left\{F(\bullet \bullet) \mathcal{P}_{-} F(\bullet \bullet)-\mathcal{P}_{-} F(\bullet \bullet) \mathcal{P}_{-} F(\bullet \bullet)\right\}=0,
$$

так как $F(\bullet \bullet-)$ является $\mathcal{P}$-отрицательным.

Теперь уже просто понять, каким образом происходят сокращения: для каждого конкретного графа все деревья можно разбить на пары, и единственное отличие между деревьями в паре заключается в наличии внешнего прямоугольника или, на языке формулы $(5), \gamma_{0}=\gamma_{1}=\Gamma_{1}$. Из-за наличия дополнительного проектора $\left(-\mathcal{P}_{-}\right)$ соответствующие графы из каждой пары точно сокращают друг друга.

В дальнейшем мы не будем приводить примеров применения формулы лесов второго уровня в случаях, когда она приводит к очевидному ответу, поскольку можно применить такие же рассуждения, как в этом пункте, для доказательства сокращения вкладов от большинства графов.

I.1.4. Проектор общего вида. Далее, обобщая наши рассуждения, мы не фиксируем конкретную зависимость от $\epsilon$ коэффициентов $A(\epsilon)$ и $B(\epsilon)$ в

$$
F(T)=A(\epsilon) T+B(\epsilon) T^{2}=A(\epsilon) \bullet+B(\epsilon) \bullet \bullet,
$$

T.e.

$$
\widehat{F}(\bullet)=A(\epsilon), \quad \widehat{F}(\bullet \bullet)=B(\epsilon)
$$

(например, это могут быть произвольные ряды Лорана), а также конкретный выбор проектора $\mathcal{P}_{-}$(к примеру, он может выбирать слагаемые вида $\epsilon^{k}$ с $k>k_{0}$ или слагаемые типа $\epsilon^{k} \operatorname{sh} \epsilon$ ). Уравнение (4) применимо во всех случаях! На самом деле контрчленами будут

$$
A(\epsilon) Q(\bullet)=-\mathcal{P}_{-}\{F(\bullet-)\}=-A_{-},
$$


T.e.

$$
\begin{aligned}
Q(\bullet-) & =-\frac{A_{-}}{A}, \\
A(\epsilon) Q(\bullet-) & =-\mathcal{P}_{-}\left\{F(\bullet \bullet)\left(1+2 Q(\bullet)+[Q(\bullet-)]^{2}\right)\right\}= \\
& =-\left[B\left(1-\frac{A_{-}}{A}\right)^{2}\right]_{-}=-\left(\frac{B A_{+}^{2}}{A^{2}}\right)_{-}, \\
Q[3] & =Q(\bullet \bullet \bullet)=-\frac{1}{A} \mathcal{P}_{-}\{F[2](2 Q[2]+2 Q[2] Q[1])\}= \\
& =\frac{2}{A}\left(B \frac{1}{A}\left(\frac{B A_{+}^{2}}{A^{2}}\right)_{-}\left\{1-\frac{A_{-}}{A}\right\}\right)_{-}=\frac{2}{A}\left(\frac{B A_{+}}{A^{2}}\left(\frac{B A_{+}^{2}}{A^{2}}\right)_{-}\right)_{-}, \\
Q[4] & =-\frac{1}{A} \mathcal{P}_{-}\left(F[2] Q^{2}[2]+2 F[2] Q[3]+2 F[2] Q[3] Q[1]\right)= \\
& =-\frac{1}{A}\left(\frac{B}{A^{2}}\left(\frac{B A_{+}^{2}}{A^{2}}\right)_{-}^{2}+\frac{4 B A_{+}}{A^{2}}\left(\frac{B A_{+}}{A^{2}}\left(\frac{B A_{+}^{2}}{A^{2}}\right)_{-}\right)_{-},\right.
\end{aligned}
$$

Поэтому

$$
\begin{aligned}
\widetilde{T}=A_{+} & \frac{T}{A}-\left(\frac{B A_{+}^{2}}{A^{2}}\right)_{-} \frac{T^{2}}{A}+2\left(\frac{B A_{+}}{A^{2}}\left(\frac{B A_{+}^{2}}{A^{2}}\right)_{-}\right)_{-} \frac{T^{3}}{A} \\
& -\left\{4\left(\frac{B A_{+}}{A^{2}}\left(\frac{B A_{+}}{A^{2}}\left(\frac{B A_{+}^{2}}{A^{2}}\right)_{-}\right)_{-}\right)_{-}+\left(\frac{B}{A^{2}}\left(\frac{B A_{+}^{2}}{A^{2}}\right)_{-}^{2}\right)_{-}\right\} \frac{T^{4}}{A}+\cdots
\end{aligned}
$$

и

$$
\begin{aligned}
F(\widetilde{T})=A \widetilde{T} & +B \widetilde{T}^{2}=A_{+} T+\left(\frac{B A_{+}^{2}}{A^{2}}-\left(\frac{B A_{+}^{2}}{A^{2}}\right)_{-}\right) T^{2}- \\
& -2\left[\frac{B A_{+}}{A^{2}}\left(\frac{B A_{+}^{2}}{A^{2}}\right)_{-}-\left(\frac{B A_{+}}{A^{2}}\left(\frac{B A_{+}^{2}}{A^{2}}\right)_{-}\right)_{-}\right]^{3}+ \\
& +\left\{4 \frac { B A _ { + } } { A ^ { 2 } } ( \frac { B A _ { + } } { A ^ { 2 } } ( \frac { B A _ { + } ^ { 2 } } { A ^ { 2 } } ) _ { - } ) _ { - } 4 \left(\frac{B A_{+}}{A^{2}}\left(\frac{B A_{+}}{A^{2}}\left(\frac{B A_{+}^{2}}{A^{2}}\right)_{-}\right)_{-}+\right.\right. \\
& \left.+\frac{B}{A^{2}}\left(\frac{B A_{+}^{2}}{A^{2}}\right)_{-}^{2}-\left(\frac{B}{A^{2}}\left(\frac{B A_{+}^{2}}{A^{2}}\right)_{-}^{2}\right)_{-}\right\}^{4}+\cdots
\end{aligned}
$$

Очевидно, что все слагаемые перед каждой степенью $T$ собираются в $\mathcal{P}$-положительное выражение:

$$
\begin{aligned}
F(\widetilde{T})=A_{+} T+\left(\frac{B A_{+}^{2}}{A^{2}}\right)_{+} T^{2}-2\left[\frac{B A_{+}}{A^{2}}\left(\frac{B A_{+}^{2}}{A^{2}}\right)_{-}\right]_{+}^{3} T^{3} \\
+\left[4 \frac{B A_{+}}{A^{2}}\left(\frac{B A_{+}}{A^{2}}\left(\frac{B A_{+}^{2}}{A^{2}}\right)_{-}\right)_{-}+\frac{B}{A^{2}}\left(\frac{B A_{+}^{2}}{A^{2}}\right)_{-}^{2}\right]_{+} T^{4}+\cdots
\end{aligned}
$$

Правая часть здесь содержит лишь чистые $(+)$-проекции, однако следует отметить, что в случае общего положения перенормированная функция $F(\widetilde{T})$ не является квадратичной по $T$ в отличие от исходной $F(T)$. 
I.1.4.1. Конкретный пример. Чтобы пояснить последнее утверждение, рассмотрим простой пример. Пусть $\mathcal{P}_{-}$выбирает лишь слагаемые порядка $\epsilon, \mathcal{P}_{-} F=$ $(\partial F / \partial \epsilon)_{\epsilon=0}$ и

$$
F(T)=T+b \epsilon T^{2}+c \epsilon^{2} T^{3}
$$

Для такой $F(T)$ мы можем легко проверить, что есть только одно ненулевое $Q$, а именно $Q(\bullet \bullet)=-b \epsilon$. Отсюда следует, что $\widetilde{T}=T-b \epsilon T^{2}$. Поэтому перенормированная $F(T)$ не является полиномом второй степени по $T$ :

$$
\begin{aligned}
F(T) & =F(\widetilde{T}(T))=T-b \epsilon T^{2}+b \epsilon\left(T-b \epsilon T^{2}\right)^{2}+c \epsilon^{2}\left(T-b \epsilon T^{2}\right)^{3}= \\
& =T+\left(c-2 b^{2}\right) \epsilon^{2} T^{3}+\left(b^{3}-3 c b\right) \epsilon^{3} T^{4}+3 c b^{2} \epsilon^{4} T^{5}-c b^{3} \epsilon^{5} T^{6} .
\end{aligned}
$$

Это, казалось бы, противоречит всем предыдущим примерам: нам следовало бы ожидать что $F(\widetilde{T})=T+c \epsilon^{2} T^{3}$. Однако отличие заключается в том, что $Q$, которое перенормировало бы $F$ таким способом, должно содержать $\mathcal{P}$-положительные слагаемые и не может быть получено из формулы лесов. Формула лесов дает $Q(\Gamma)$, удовлетворяющее условию (2).

Это первый пример, показывающий что перенормированная $F$ может сильно отличаться от исходной функции - быть значительно сложнее или, наоборот, проще. Это значит, что формула лесов (5), непосредственно описывающая изменение вида функции, не является тривиальной. Посмотрим, как получается результат (18) из формулы лесов второго уровня.

Для $F(\bullet \bullet-)$ мы получим такой же результат, как и в $(15)$. Для $F(\bullet \bullet \bullet-)$ имеем (cp. c (16))

$$
\begin{array}{rl}
F_{\mathrm{R}}(\bullet \bullet-)= & F(\bullet \bullet-)+ \\
& +2\left\{F(\bullet \bullet) \frac{1}{F(-\bullet)}\left(-\mathcal{P}_{-}\right) F(\bullet \bullet)+\right. \\
& \left.+F(\bullet) \frac{1}{F(-)}\left(-\mathcal{P}_{-}\right)\left[F(\bullet \bullet) \frac{1}{F(-)}\left(-\mathcal{P}_{-}\right) F(\bullet \bullet)\right]\right\}= \\
=c & c \epsilon^{2}+2\left\{b \epsilon\left(-\mathcal{P}_{-}\right) b \epsilon+\left(-\mathcal{P}_{-}\right)\left[b \epsilon\left(-\mathcal{P}_{-}\right) b \epsilon\right]\right\}
\end{array}
$$

при этом $\left(-\mathcal{P}_{-}\right)\left[b \epsilon\left(-\mathcal{P}_{-}\right) b \epsilon\right]=0$, и мы получаем $F_{\mathrm{R}}(\bullet \bullet-)=c \epsilon^{2}-2 b^{2} \epsilon^{2}$. Аналогичным образом воспроизводятся коэффициенты перед $T^{4}, T^{5}, T^{6}$. Любопытно проследить, каким образом $F_{\mathrm{R}}(\Gamma)$ обращается в нуль для линейных графов с более чем шестью вершинами, как это предсказывает формула (18). Это также почти очевидно: легко заметить, что деревья с вложенными прямоугольными подграфами не дают вклада: $\mathcal{P}_{-}$в $(5)$ действует вверх вдоль ветвей дерева, а $\mathcal{P}_{-} \epsilon^{n}=0$ для $n>1$. Все наши прямоугольники могут содержать только две вершины, и $F(\Gamma)$ принимает ненулевое значение только для линейных графов с одной, двумя или тремя вершинами. Умножение 3 на 2 дает 6 , и для всех линейных графов с более чем шестью вершинами $F_{\mathrm{R}}(\Gamma)$ обращается в нуль, что соответствует (18). 
I.1.4.2. Другой пример. Чтобы еще более углубить наше понимание задач, которые решаются с помощью формулы лесов, рассмотрим еще один пример с таким же проектором, как и в предыдущем п. I.1.4.1, но с другим выбором функции

$$
F(T)=\frac{1}{\epsilon} T+b \epsilon T^{2} .
$$

В этом случае имеется один неисчезающий контрчлен $Q(\Gamma)$ :

$$
\frac{1}{\epsilon} Q(\bullet \bullet)=-\mathcal{P}_{-}\{F(\bullet \bullet)\}=-\mathcal{P}_{-}\{b \epsilon\}=-b \epsilon, \quad Q(\bullet \bullet)=-b \epsilon^{2} .
$$

Мы видим что $\mathcal{P}_{+} Q(\bullet \bullet-)=-b \epsilon^{2} \neq 0$, и это противоречит условию (2). На этом примере видно отличие настоящего условия от использованного в $(2)$, что упомянуто в сноске 1: $F(\bullet) Q(\bullet \bullet)$, а не просто $Q(\bullet \bullet-)$ должно быть $\mathcal{P}$-отрицательным. И в нашем случае это действительно выполняется: $\mathcal{P}_{+} F(\bullet-) Q(\bullet \bullet-)=-\mathcal{P}_{+} b \epsilon=0$.

\section{I.1.5. Петлевые диаграммы, необходимость введения вакуумной энер-} гии: $F(T) \rightarrow F(v, T)=v+T+\epsilon T^{2}$. Давайте теперь представим $T$ и $T^{2}$ двумя петлевыми диаграммами $\bigcirc$ и $\bigcirc$. Однако для этих диаграмм $\Gamma / \Gamma=\bullet$ есть вершина нулевой валентности (без внешних линий), которая не представлена в нашей функции $F(T)$. Следовательно, для того чтобы применить формулу (4) в данной ситуации, нам следует ввести дополнительное слагаемое в $F$, связанное с данной вершиной. Если мы обозначим соответствующую константу связи $v$, наша модифицированная функция может быть записана как

$$
F(v, T)=v+T+\epsilon T^{2}=\bullet+\bigcirc+\epsilon \bullet .
$$

Теперь мы можем применить (4), и единственным неисчезающим $Q(\Gamma)$ будет

$$
Q(\bigcirc)=-\mathcal{P}_{-}\{F(\bigodot)\}=-\epsilon .
$$

Так как поправки к $v$ и к $T$ отвечают графам без внешних ног и с двумя внешними ногами соответственно, уравнение (20) дает сдвиг только $v$ :

$$
\tilde{v}=v+Q(\bigcirc)=v-\epsilon T^{2}, \quad \widetilde{T}=T,
$$

так что перенормированная функция $F(\tilde{v}, \widetilde{T})=\tilde{v}+T+\epsilon T^{2}=v+T$ в действительности не зависит от $\epsilon$.

I.1.6. Смешанные цепочечно-петлевые диаграммы, восстановление тензорной структуры. Теперь представим $T$ петлевой диаграммой $\bigcirc$, а $T^{2}$ зададим в виде цепочечной диаграммы $\bullet \bullet$. В данном случае есть две константы связи,

$$
F(v, T)=\alpha v+(a+b) T+\epsilon c T^{2}=\alpha \bullet+a \bullet+b \bigcirc+\epsilon c \bullet \bullet .
$$

Теперь из (4) мы получаем

$$
a Q(\bullet \bullet)=-\mathcal{P}_{-}\{F(\bullet \bullet)\}=-\epsilon c,
$$




$$
\begin{aligned}
\alpha Q(\bullet) & =-\mathcal{P}_{-}\{F(\bigcirc) Q(\bullet \bullet)\}=-b\left(-\frac{\epsilon c}{\alpha}\right)=\frac{\epsilon b c}{\alpha} \\
a Q(\bullet \bullet-) & =-\mathcal{P}_{-}\{2 Q(\bullet \bullet) F(\bullet \bullet)\}=2 \frac{(\epsilon c)^{2}}{a} \\
\alpha Q(\bullet) & =-\mathcal{P}_{-}\{F(\bigcirc) Q(\bullet \bullet \bullet)\}=-\frac{b}{\alpha} \mathcal{P}_{-}\{Q(\bullet \bullet \bullet)\}=-2 \frac{(\epsilon c)^{2}}{a} \frac{b}{\alpha}
\end{aligned}
$$

Чтобы понять, почему коэффициенты 2, 3 (равные числу способов, которыми можно выбрать соответствующий цепочечный граф из петлевого) не появляются в (24) и (26), следует восстановить тензорные структуры вершин $v$ и $T$. После того как мы это сделаем, задача примет следующий вид:

$$
\alpha \tilde{v}+a \widetilde{T}^{i j}+b \widetilde{T}^{l l}+\epsilon c \widetilde{T}^{i l} \widetilde{T}^{l j}=\alpha v+a T^{i j}+b T^{l l} .
$$

Из-за наличия двух различных тензорных структур данное соотношение является не одним уравнением, а системой

$$
\begin{aligned}
& a \widetilde{T}^{i j}+\epsilon c \widetilde{T}^{i l} \widetilde{T}^{l j}=a T^{i j}, \\
& \alpha \tilde{v}+b \widetilde{T}^{l l}=\alpha v .
\end{aligned}
$$

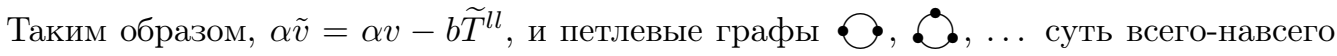
соответствующие цепочечные диаграммы $\rightarrow \bullet-,-\bullet \bullet-, \ldots$ со склеенными концами.

Для перенормировки $v$ и $T$ мы получаем

$$
\begin{aligned}
& \tilde{v}=\bullet+Q(\bigcirc) \bigcirc+Q(\text { ठ) }) \cdot+\cdots=v+\frac{\epsilon c b}{a \alpha} T^{2}-2 \frac{(\epsilon c)^{2} b}{a \alpha} T^{3}+\cdots \\
& \tilde{T}=\bullet+Q(\bullet \bullet) \bullet \bullet+Q(\bullet \bullet \bullet) \bullet \bullet \bullet+\cdots=T-\frac{\epsilon c}{a} T^{2}+2\left(\frac{\epsilon c}{a}\right)^{2} T^{3}-\cdots
\end{aligned}
$$

Поскольку значения $Q\left[n^{\text {(loop) }}\right]$ на петлевых диаграммах определяются значениями $Q[n]$ на соответствующих цепочечных диаграммах, мы можем выполнить суммирование с помощью производящей функции так же, как это было сделано в (11):

$$
\begin{aligned}
F(\tilde{v}, \widetilde{T})=\alpha \tilde{v} & +(a+b) \widetilde{T}+\epsilon c \widetilde{T}^{2}=\alpha\left(v+\frac{\epsilon b c}{a \alpha} T^{2}+\cdots\right)+ \\
& +(a+b)\left(T-\frac{\epsilon c}{a} T^{2}+\cdots\right)+\epsilon c\left(T-\frac{\epsilon c}{a} T^{2}+\cdots\right)^{2}=\alpha v+(a+b) T .
\end{aligned}
$$

Стоит отметить, что даже если бы $\alpha$ изначально было равно нулю, оно в процессе перенормировки приобрело бы не зависящий от $\alpha$ сдвиг. Вершина нулевой валентности автоматически генерируется формулой лесов. Однако это происходит сингулярным образом: не зависящая от $\alpha$ поправка сначала делится, а потом умножается на $\alpha \rightarrow 0$. 


\section{I.2. МНОГОЧЛЕН ОБЩЕГО ВИДА ИЗ ДВУХВАЛЕНТНОЙ ВЕРШИНЫ}

Теперь мы приступим к решению более общей проблемы, чем в предыдущих разделах: выберем в качестве $F(T)$ сумму двух многочленов, а проектор возьмем тот же, что и в (7): пусть

$$
F(T)=R(T)+\epsilon G(T) .
$$

Проблема перенормировки сводится к уравнению

$$
R(\widetilde{T})+\epsilon G(\widetilde{T})=R(T)
$$

и решение представляется в виде степенного ряда по $\epsilon$ :

$$
\widetilde{T}=T-\epsilon \frac{G(T)}{R^{\prime}(T)}+\epsilon^{2} \frac{G(T)}{2\left(R^{\prime}(T)\right)^{3}}\left(2 R^{\prime}(T) G^{\prime}(T)-R^{\prime \prime}(T) G(T)\right)+\cdots
$$

Возникает очевидный вопрос: каково происхождение различных степеней $R^{\prime}(T)$ с точки зрения формулы лесов.

I.2.1. Простейшая нетривиальная функция $F(T)=T+\alpha T^{2}+\epsilon T^{3}$. Мы начнем с самой простой нетривиальной функции $R$ : пусть $R(T)=T+\alpha T^{2}$ и $G(T)=$ $\epsilon T^{3}$. Подставляя эти формулы в (28), мы получаем

$$
\widetilde{T}=T-\epsilon \frac{T^{3}}{1+2 \alpha T}+\epsilon^{2} \frac{T^{5}(3+5 \alpha T)}{(1+2 \alpha T)^{3}}+\cdots .
$$

После разложения знаменателей по $\alpha T$ имеем

$$
\widetilde{T}=T-\epsilon\left(T^{3}-2 \alpha T^{4}+4 \alpha^{2} T^{5}-8 \alpha^{3} T^{6}+\cdots\right)+\epsilon^{2}\left(3 T^{5}-13 \alpha T^{6}+42 \alpha^{2} T^{7}+\cdots\right)+\cdots
$$

Приступим к решению данной задачи, пользуясь формулой лесов:

$$
\begin{aligned}
F(T) & =T+\alpha T^{2}+\epsilon T^{3}=\bullet+\alpha \bullet \bullet+\epsilon \bullet \bullet- \\
Q[3] & =Q(\bullet \bullet \bullet-)=-\epsilon, \\
Q[4] & =-\mathcal{P}_{-}(2 F[2] Q[3])=2 \alpha \epsilon, \\
Q[5] & =-\mathcal{P}_{-}(2 F[2] Q[4]+3 F[3] Q[3])=3 \epsilon^{2}-4 \alpha^{2} \epsilon, \\
Q[6] & =-\mathcal{P}_{-}\left(F[2] Q^{2}[3]+2 F[2] Q[5]+3 F[3] Q[4]\right)=8 \alpha^{3} \epsilon-13 \alpha \epsilon^{2}
\end{aligned}
$$

Таким образом, для $\widetilde{T}$ мы получаем

$$
\begin{aligned}
\widetilde{T} & =T-\epsilon T^{3}+2 \alpha \epsilon T^{4}+\left(3 \epsilon^{2}-4 \alpha^{2} \epsilon\right) T^{5}+\left(8 \alpha^{3} \epsilon-13 \alpha \epsilon^{2}\right) T^{6}+\cdots= \\
& =T-\epsilon\left(T^{3}-2 \alpha T^{4}+4 \alpha^{2} T^{5}-8 \alpha^{3} T^{6}+\cdots\right)+\epsilon^{2}\left(3 T^{5}-13 \alpha T^{6}+\cdots\right)+\cdots,
\end{aligned}
$$

т.е. мы имеем выражение (30), но разложенное сначала по $\alpha T$, а потом по $\epsilon$.

Полезно попытаться просуммировать явно все слагаемые, которые дают вклад в первом и втором порядках по $\epsilon$. Как обычно, это может быть сделано с помощью 
производящих функций $Q_{(1)}(t)=\sum_{n=3} t^{n} Q_{(1)}[n]$ и $Q_{(2)}(t)=\sum_{n=3} t^{n} Q_{(2)}[n]$, где $Q_{(1)}[n], Q_{(2)}[n]$ означают вклады в $Q[n]$ порядка $\epsilon, \epsilon^{2}$, соответственно.

Поскольку $Q[n]$ не содержит слагаемых порядка $\epsilon^{0}$, только члены с одним прямоугольным подграфом дают вклад в $Q_{(1)}[n+1]$ :

$$
Q_{(1)}[n+1]=-\mathcal{P}_{-}\left(2 F[2] Q_{(1)}[n]\right)
$$

Для $Q_{(1)}(t)$ это дает

$$
Q_{(1)}(t)=Q[3] t^{3}-2 \alpha t Q_{(1)}(t), \quad Q_{(1)}(t)=\frac{t^{3} Q[3]}{1+2 \alpha t}=-\epsilon \frac{t^{3}}{1+2 \alpha t} .
$$

Для $Q_{(2)}[n+1]$ мы должны учесть такие три вклада: $F[2] Q_{(2)}[n], F[3] Q_{(1)}[n]$ и $F[2] Q_{(1)}[n] Q_{(1)}[k]$. Получаем

$Q_{(2)}[n+3]=-\mathcal{P}_{-}\left(2 F[2] Q_{(2)}[n+2]+3 F[3] Q_{(1)}[n+1]+F[2] \sum_{k=3}^{n} Q_{(1)}[k] Q_{(1)}[n+3-k]\right)$.

Таким образом, $Q_{(2)}(t)$ удовлетворяет уравнению

$$
Q_{(2)}(t)=-\left(2 t F[2] Q_{(2)}(t)+3 t^{2} F[3] Q_{(1)}(t)+F[2] Q_{(1)}^{2}(t)\right),
$$

следовательно,

$$
Q_{(2)}(t)=-\frac{3 \epsilon t^{2} Q_{(1)}(t)+\alpha Q_{(1)}^{2}(t)}{1+2 \alpha t}=\frac{\epsilon^{2} t^{5}(3+5 \alpha t)}{(1+2 \alpha t)^{3}}
$$

Как видно, после пересуммирования мы воспроизвели (29).

I.2.2. Функция $F(T)$ общего вида. Чтобы понять, каким образом формула лесов может дифференцировать многочлены и давать $R^{\prime}(T)$ в знаменателе в формуле (28), необходимо просуммировать слагаемые порядка $\epsilon^{1}$. Точно так же, как в предыдущем пункте, мы выполним это путем решения уравнения на производящую функцию:

$$
F(T)=T+\sum_{n=2}^{R} r_{n} T^{n}+\epsilon\left(\sum_{n=1}^{G} g_{n} T^{n}\right)
$$

Здесь $R$ и $G$ обозначают степени многочленов $R(T)$ и $G(T)$, соответственно, и для простоты положено $r_{1}=1$. Если

$$
Q_{(1)}(t)=\sum_{n=1} t^{n} Q_{(1)}[n]
$$

то для $n \geqslant R$

$$
\begin{aligned}
Q_{(1)}[n]=- & \mathcal{P}_{-}^{(1)}\left\{2 F[2] Q_{(1)}[n-1]+3 F[3] Q_{(1)}[n-2]+\right. \\
& \left.+4 F[4] Q_{(1)}[n-3]+\cdots+R F[R] Q_{(1)}[n-R+1]\right\}
\end{aligned}
$$


где $\mathcal{P}_{-}^{(1)}$ - проектор, который выбирает только слагаемые порядка $\epsilon^{1}$. Коэффициенты $2,3, \ldots, R$ дают число способов, которыми мы можем вырезать прямоугольный подграф соответствующей длины из цепочечного графа $[n]$. Если записать это соотношение через $Q_{(1)}(t)$, то получится

$$
Q_{(1)}(t)=-\left(2 r_{2} t+3 r_{3} t^{2}+\cdots+R r_{\mathrm{R}} t^{R-1}\right) Q_{(1)}(t)-\left(g_{1} t-g_{2} t^{2}-\cdots-g_{G} t^{G}\right)
$$

и окончательно

$$
Q_{(1)}(t)=-\frac{G(t)}{R^{\prime}(t)} .
$$

Аналогичным образом мы можем провести суммирование в каждом порядке по $\epsilon$.

\section{I.3. КУБИЧЕСКОЕ УРАВНЕНИЕ. ТРЕХВАЛЕНТНАЯ ВЕРШИНА И ПЕТЛИ}

Пусть $T$ соответствует трехвалентной вершине. Тогда мы можем использовать формулу лесов для решения кубического уравнения $\widetilde{T}+\epsilon \widetilde{T}^{3}=T$, предполагая, что проектор задается в формуле (7). Решением является

$$
\widetilde{T}=T-\epsilon T^{3}+3 \epsilon^{2} T^{5}-12 \epsilon^{3} T^{7}+55 \epsilon^{4} T^{9}+\cdots=T+\sum_{n=1}(-1)^{n} c_{n} \epsilon^{n} T^{2 n+1},
$$

где $c_{n}$ - это число трехвалентных деревьев с корнем с $n$ вершинами, т.е.

$$
c_{1}=1 \longleftrightarrow c_{2}=3 \longleftrightarrow \underbrace{}_{+}
$$

Мы получим это решение из формулы лесов. Как в случае двухвалентной вершины, имеются различные возможности представления функции $F(T)$ и, таким образом, различные реализации процедуры перенормировки.

I.3.1. Первая реализация. Представить $T+\epsilon T^{3}$ можно, например, так:

$$
F(T)=T+\epsilon T^{3}=\ldots+\epsilon
$$

Когда мы начинаем вычислять $Q(\Gamma)$, формула лесов сама выбирает существенные графы из большого множества всевозможных графов с трехвалентными вершинами. Имеем

$$
\begin{aligned}
Q(\longrightarrow) & =-\mathcal{P}_{-}\{F(\longrightarrow)\}=0, \\
Q(Z) & =-\mathcal{P}_{-}\{F(\longrightarrow)=-\epsilon,
\end{aligned}
$$



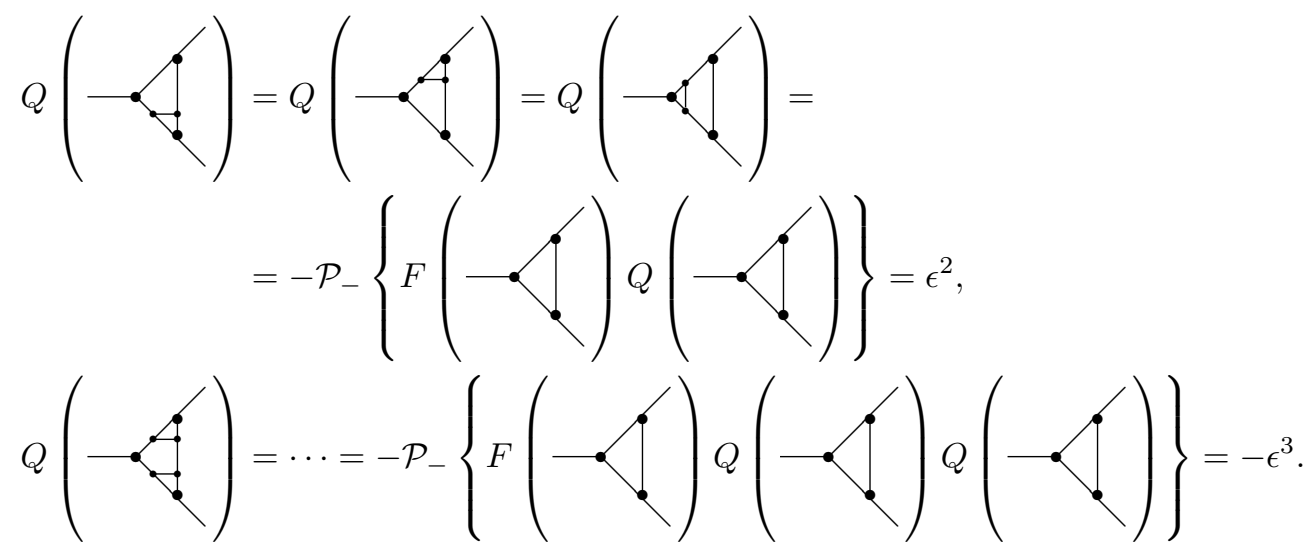

Легко заметить что, так как вложенные графы не дают вклада в формулу лесов первого уровня, все неисчезающие $Q(\Gamma)$ будут иметь вид

$$
Q(\Gamma)=(-1)^{(k-1) / 2} \epsilon^{(k-1) / 2},
$$

где $k$ - это число вершин в Г. Поэтому проблема сводится к подсчету числа графов в порядке $k$, дающих ненулевой вклад. Это, однако, становится простой задачей, если заметить, что существует взаимно однозначное соответствие между "треугольными" графами и деревьями с корнем из (31):

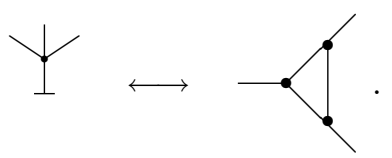

Когда мы добавляем три ребра в какую-то вершину исходного дерева, это отвечает вставке в соответствующую вершину нашего графа, к примеру

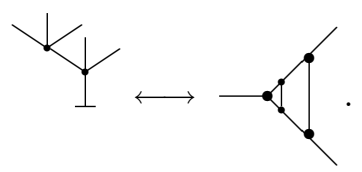

Поэтому из формулы лесов мы получим следующий результат для $\widetilde{T}$ :

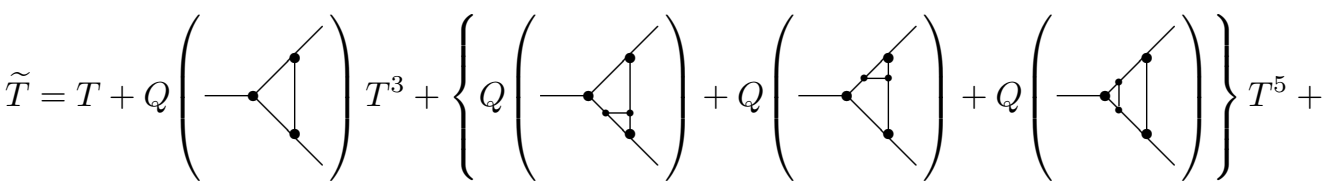

$$
\begin{aligned}
& +\{Q(\vdots)+\cdots\} T^{7}+\cdots=T+\sum_{n=1}(-1)^{n} c_{n} \epsilon^{n} T^{2 n+1} \text {. }
\end{aligned}
$$


I.3.2. Вторая реализация. Эту же функцию $F(T)$ можно представить в другом виде:

$$
F(T)=T+\epsilon T^{3}=\longrightarrow+3 \epsilon \multimap \prec
$$

Здесь мы вставили коэффициент 3, который отражает симметрию задачи. Его появление становится очевидным, если мы восстановим индексы в тройной симметричной по всем индексам вершине: $T \rightarrow T_{i j k}$, так что теперь (по повторяющимся индексам подразумевается суммирование)

$F_{i j k}(T)=T_{i j k}+\epsilon\left((T T)_{i m} T_{m j k}+(T T)_{j m} T_{i m k}+(T T)_{k m} T_{i j m}\right), \quad(T T)_{i m}=T_{i p q} T_{m p q}$,

и на самом деле существуют три различные однопетлевые диаграммы.

Первыми контрчленами будут

$$
Q\left(\longrightarrow \cdot\langle)=-\mathcal{P}_{-}\{F(\longrightarrow \bigcirc<)\}=-\epsilon .\right.
$$

Здесь слева стоит та же диаграмма, что и в правой части равенства, поэтому коэффициент 3 не возникает.

В следующем порядке имеются диаграммы трех различных топологий:

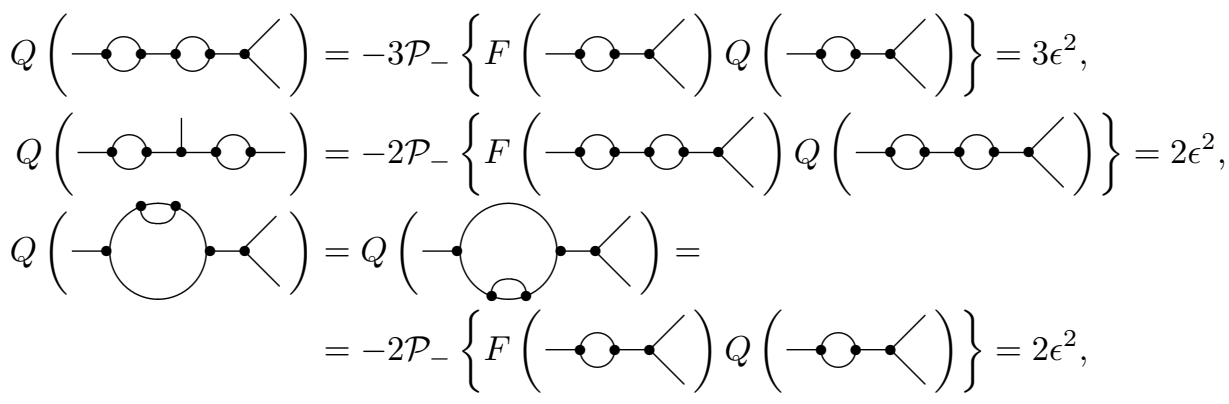

где коэффициенты 3 в (36) и 2 в (37) и (38) соответствуют числу различных прямоугольных подграфов.

Учитывая три различные ориентации диаграмм, для контрчлена $Q(T)$ мы получим

$$
\widetilde{T}=T-3 \epsilon T^{3}+3 \cdot(3+2+2 \times 2) \epsilon^{2} T^{5}+\cdots
$$

и

$$
F(\widetilde{T})=\widetilde{T}+3 \epsilon \widetilde{T}^{3}=\left(T-3 \epsilon T^{3}+27 \epsilon^{2} T^{5}+\cdots\right)+3 \epsilon\left(T-3 \epsilon T^{3}+27 \epsilon^{2} T^{5}+\cdots\right)^{3}=T .
$$

\section{I.4. КУБИЧЕСКОЕ УРАВНЕНИЕ. ДВУХ- И ТРЕХВАЛЕНТНАЯ ВЕРШИНЫ}

Мы уже сталкивались с задачей, в которой присутствовала больше чем одна константа связи, но наши примеры были довольно простыми. В этом разделе мы попытаемся показать специфику формулы лесов при работе с несколькими константами 
связи. Рассмотрим

$$
F(T)=T_{2}+\epsilon_{2} T_{3}^{2}+T_{3}+\epsilon_{3} T_{3}^{3}+3 \epsilon_{3}^{2} T_{3}^{3} T_{2}=
$$

пусть $\mathcal{P}_{-}$выбирает все слагаемые вида $\epsilon_{2}^{\alpha} \epsilon_{3}^{\beta}$ с $|\alpha|+|\beta| \geqslant 1$.

Опять-таки, как и в п. I.1.6, из-за наличия различных тензорных структур уравнение $F(\widetilde{T})=T_{2}+T_{3}$ подразумевает перенормировку двух различных функций:

$$
\widetilde{T}_{2}+\epsilon_{2} \widetilde{T}_{3}^{2}=T_{2}, \quad \widetilde{T}_{3}+\epsilon_{3} \widetilde{T}_{3}^{3}+3 \epsilon_{3}^{2} \widetilde{T}_{3}^{3} \widetilde{T}_{2}=T_{3}
$$

Однако на этот раз два уравнения взаимосвязанны, и в результате итерационной процедуры мы должны находить $\widetilde{T}_{2}$ и $\widetilde{T}_{3}$ одновременно. Попробуем решить данную задачу при помощи формулы лесов. Для первого графа все просто:

$$
Q(\multimap-)=-\mathcal{P}_{-}\{F(\multimap-)\}=-\epsilon_{2} .
$$

Однако когда мы посмотрим на следующий граф с двумя внешними концами, возникает такое затруднение: неизвестно, как найти

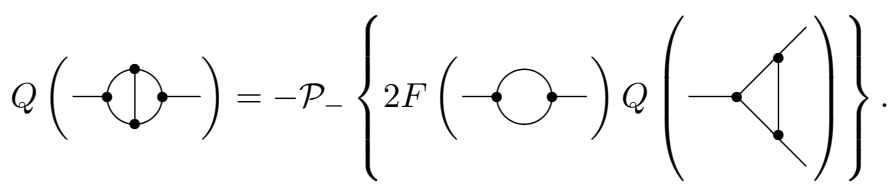

Оказывается, что сначала нам надо вычислить

$$
Q\left(\{)=-\mathcal{P}_{-}\{F()\}=-\epsilon_{3}\right.
$$

и только потом вернуться к

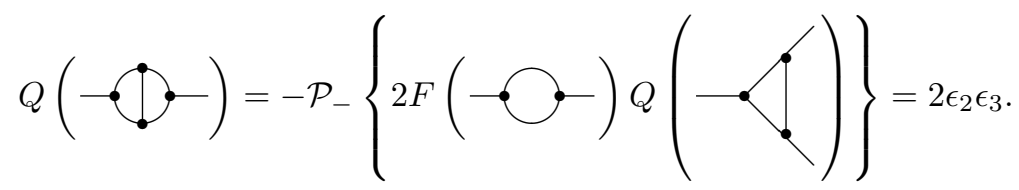

Для следующих графов имеем

$$
Q(\vdots)=-\mathcal{P}_{-}\{F(\vdots),
$$




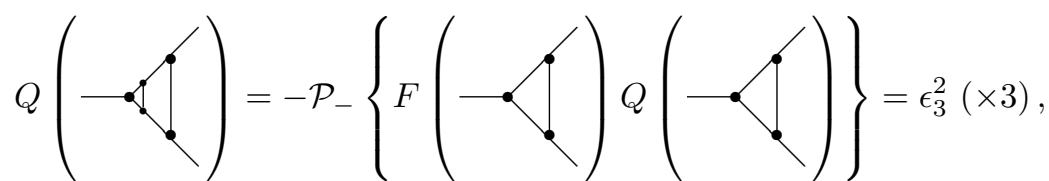

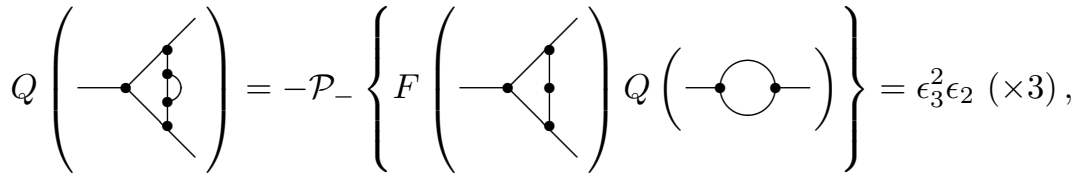

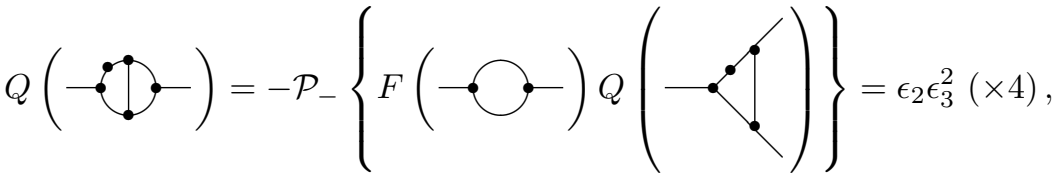

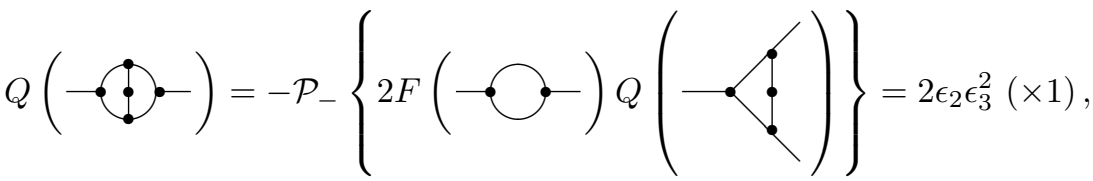

где $(\times 3),(\times 4), \ldots$ означает число возможных положений точки или, в случае $(44)$, число ориентаций соответствующего графа. Снова заметим, что для вычисления (45) нам надо знать (40), для (46) и (47) необходимо (43), хотя эти графы имеют разное число внешних линий.

Формула лесов сама выбирает диаграммы, которые дают вклад в перенормировку. Именно благодаря связи между процессами перенормировки $T_{2}$ и $T_{3}$ невозможно сначала найти $Q$ для диаграмм с двумя внешними концами, а потом для диаграмм с тремя концами: это следует делать одновременно, порядок за порядком. Итак, мы получаем

$$
\begin{aligned}
& \widetilde{T}_{2}=T_{2}-\epsilon_{2}{\stackrel{(40)}{T_{3}^{2}}}^{2}+2 \epsilon_{2} \epsilon_{3} T_{3}^{4}+4 \epsilon_{2} \epsilon_{3}^{2} \stackrel{(46)}{T}_{2} T_{3}^{4}+2 \epsilon_{2} \epsilon_{3}^{2}{ }^{(47)} T_{2}^{4}+\cdots, \\
& \widetilde{T}_{3}=T_{3}-\epsilon_{3} T_{3}^{3}-3 \epsilon_{3}^{2} \stackrel{(43)}{T}_{2} T_{3}^{3}+3 \epsilon_{3}^{2} T_{3}^{(44)}+3 \epsilon_{2} \epsilon_{3}^{2} T_{3}^{(45)}+\cdots .
\end{aligned}
$$

Чтобы сделать понятным происхождение всех слагаемых, над каждым из них написан номер формулы, которая соответствует данному вкладу. Легко проверить, что (48), (49) дают решение исходной задачи.

\section{II. ПРИМЕРЫ ИЗ КВАНТОВОЙ ТЕОРИИ ПОЛЯ}

Квантовая теория поля - это естественная область применения формулы лесов, изначально созданной именно для этого. В теории возмущений в квантовой теории поля часто возникают расходимости, связанные с интегралами по импульсам в петлевых диаграммах, т.е. возникающие из суммы по тензорным индексам в разложении по диаграммам. Чтобы придать смысл расходящимся интегралам, используется регуляризация, которая приводит к зависимости от дополнительного параметра (типа параметра ультрафиолетового обрезания $\Lambda$, параметра $\epsilon=4-d$ при размерной 
регуляризации или от масс $M_{i}$ в регуляризации Паули-Вилларса). В конце вычислений зависимость от этих величин должна быть устранена. Этого нельзя достичь, опустив зависящие от регуляризации вклады из конечного ответа, поскольку при этом изменятся соотношения между различными корреляторами, т.е. изменится вид статсуммы. После произвольной модификации статсумма и корреляторы не будут получаться из какого-либо функционального интеграла; таким образом, эти величины больше не будут описывать какую-либо квантово-механическую систему. Это не может произойти в случае, когда мы не меняем формы статсуммы, но при этом меняем значения ее аргументов, т.е. голых констант связи, которые в точности являются нашими параметрами $\widetilde{T}$ из предыдущих разделов. Формула лесов выражает голые константы связи $\widetilde{T}$ через физические константы связи:

$$
\widetilde{T} \equiv T+Q(T)
$$

где $Q(T)$ - сингулярные контрчлены, делающие статсумму конечной. В этом смысле формула лесов дает решение проблемы перенормировок в квантовой теории поля.

Возможность применения формулы лесов не зависит от вида и происхождения функции $F(T)$, которую мы хотим перенормировать. Единственный факт, о котором следует позаботиться, чтобы провести процесс перенормировки самосогласованно - это справедливость вершинного критерия: для каждого графа $Г$, для которого $Q(\Gamma) \neq 0$, должна существовать вершина $\Gamma / \Gamma$ в $F(T)$, т.е. $F(\Gamma / \Gamma) \neq 0$, если $Q(\Gamma) \neq 0$. Если это условие выполняется, то перенормировка в квантовой теории поля может быть произведена на уровне статсуммы, т.е. для всех корреляторов одновременно. Однако часто оказывается возможным ограничиться рассмотрением корреляторов с внешней структурой определенного типа.

\section{II.1. ТЕОРИЯ $\phi^{4}$ В ОДНОЙ И ДВУХ ПЕТЛЯХ}

Простейший нетривиальный пример - это перенормировка четырехточечного коррелятора в теории $\phi^{4}$ в двух петлях (т.е. в порядке $T^{3}$ ). Этот пример все еще является довольно простым, но тем не менее он позволяет проиллюстрировать основные свойства и специфику формулы лесов.

Рассматриваемая квантовая теория поля определяется лагранжианом

$$
L(\phi)=\frac{1}{2}\left(\partial_{\mu} \phi\right)^{2}-\frac{1}{2} m^{2} \phi^{2}-\frac{T}{4 !} \phi^{4} .
$$

Мы предполагаем, что перенормировка поля и массового слагаемого уже были проведены, и поэтому не рассматриваем перенормировку кинетического и массового членов, т.е. двухвалентные вершины и расходящиеся графы с двумя внешними 
линиями (о них см. п. 2 приложения). Мы начинаем непосредственно с четырехточечной функции, которая записывается как сумма фейнмановских диаграмм:

$$
\begin{aligned}
& F(T)=x+y+
\end{aligned}
$$

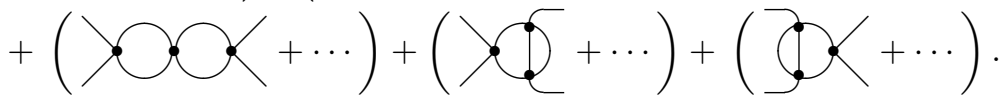

Троеточие означает опущенные диаграммы в $u$ - и $s$-каналах. В качестве $Z(\Gamma \mid T)$ мы выбираем следующие функции:

$$
\begin{gathered}
Z(\chi \mid T)=-i T, \quad Z(\text { 1-loop diagramm } \mid T)=(-i T)^{2}, \\
Z(\text { 2-loop diagramm } \mid T)=(-i T)^{3}, \quad \ldots
\end{gathered}
$$

Тогда $\widehat{F}(\Gamma)$ записываются как

$$
\begin{gathered}
F(\nearrow)=1, \\
F^{(1, t)}\left(p^{2}\right) \equiv F(\bigcirc)=\frac{1}{2} \int \frac{d^{4} k}{(2 \pi)^{4}} \frac{i}{k^{2}-m^{2}} \frac{i}{(k+p)^{2}-m^{2}} .
\end{gathered}
$$

Регуляризация Паули-Вилларса заменяет $F^{(1, t)}\left(p^{2}\right)$ на

$$
\begin{aligned}
F_{\text {reg } M}^{(1, t)}\left(p^{2}\right) & =\frac{1}{2} \int \frac{d^{4} k}{(2 \pi)^{4}}\left(\frac{i}{k^{2}-m^{2}}-\frac{i}{k^{2}-M^{2}}\right)\left(\frac{i}{(k+p)^{2}-m^{2}}-\frac{i}{(k+p)^{2}-M^{2}}\right)= \\
& =\frac{i}{32 \pi^{2}}\left\{-\ln \left(\frac{M^{2}}{\mu^{2}}\right)+\int_{0}^{1} d x \ln \frac{m^{2}-x(1-x) p^{2}}{\mu^{2}}+O\left(\frac{1}{M}\right)\right\}= \\
& =\frac{i}{32 \pi^{2}}\left\{-\ln \left(\frac{M^{2}}{\mu^{2}}\right)+I_{\text {fin }}^{(1, t)}\left(p^{2}\right)\right\} .
\end{aligned}
$$

Аналогичные формулы можно написать и для $F_{\operatorname{reg} M}^{(1, s)}$ и $F_{\operatorname{reg} M}^{(1, u)}$; здесь $p^{2}$ везде означает квадрат момента, бегущего через петлю. Расчет функции $F^{\left(2, t^{2}\right)}\left(p^{2}\right) \equiv$ $F\left(\right.$ очевиден, так как это $F^{(1, s)}$, возведенная в квадрат:

$$
F_{\mathrm{reg} M}^{\left(2, t^{2}\right)}\left(p^{2}\right)=\left(\frac{i}{32 \pi^{2}}\right)^{2}\left\{\ln ^{2}\left(\frac{M^{2}}{\mu^{2}}\right)-2 \ln \left(\frac{M^{2}}{\mu^{2}}\right) I_{\mathrm{fin}}^{(1, t)}\left(p^{2}\right)+\left(I_{\mathrm{fin}}^{(1, t)}\left(p^{2}\right)\right)^{2}\right\} .
$$

Другие двухпетлевые диаграммы более сложны, поскольку они содержат перекрывающиеся расходимости:

$$
F^{(2, t \cdot(s+u))}\left(p, p_{4}\right) \equiv F(\mathcal{C})=\int \frac{d^{4} q}{(2 \pi)^{4}} \frac{i}{q^{2}-m^{2}} \frac{i}{(p-q)^{2}-m^{2}} F^{(1, t)}\left(\left(q-p_{4}\right)^{2}\right) .
$$

Здесь $q$ - импульс, бегающий по большой петле, $p$ - суммарный импульс, проходящий через большую петлю, а $p_{4}$ - импульс, входящий в правую нижнюю ногу диаграммы. 
Подставляя $F_{\mathrm{reg} M}^{(1, t)}$ из $(52)$, мы получим

$$
\begin{aligned}
& F_{\text {reg } M}^{(2, t \cdot(s+u))}\left(p, p_{4}\right)=-2 \frac{i}{32 \pi^{2}} \ln \left(\frac{M^{2}}{\mu^{2}}\right) F_{\text {reg } M}^{(1, t)}\left(p^{2}\right)+ \\
& \quad+\frac{i}{32 \pi^{2}} \int \frac{d^{4} q}{(2 \pi)^{4}}\left(\frac{i}{q^{2}-m^{2}}-\frac{i}{q^{2}-M^{2}}\right) \times \\
& \quad \times\left(\frac{i}{(q-p)^{2}-m^{2}}-\frac{i}{(q-p)^{2}-M^{2}}\right) I_{\mathrm{fin}}^{(1, s)}\left(\left(q-p_{4}\right)^{2}\right)= \\
& =\left(\frac{i}{32 \pi^{2}}\right)^{2}\left\{2 \ln ^{2}\left(\frac{M^{2}}{\mu^{2}}\right)-2 \ln \left(\frac{M^{2}}{\mu^{2}}\right) I_{\mathrm{fin}}^{(1, s)}\left(p^{2}\right)-\ln ^{2}\left(\frac{M^{2}}{\mu^{2}}\right)+I_{\mathrm{fin}}^{(2, t \cdot(s+u))}\left(p, p_{4}\right)\right\} .
\end{aligned}
$$

Поскольку выражения для каждой из диаграмм естественно распадаются на две части, конечную в пределе $M \rightarrow \infty$ и расходящуюся, как $\ln ^{n}\left(M^{2} / \mu^{2}\right)$ с положительным целым $n$, мы определим $\mathcal{P}_{-}$так, чтобы он выбирал слагаемые вида $\ln ^{n}\left(M^{2} / \mu^{2}\right)$ c $n>0$.

Теперь мы можем применить формулу лесов (4):

$$
\begin{aligned}
Q(Y) & =Q(Y)=Q(Y)= \\
& =-\mathcal{P}_{-}\{F(Y)\}=\frac{i}{32 \pi^{2}} \ln \left(\frac{M^{2}}{\mu^{2}}\right),
\end{aligned}
$$

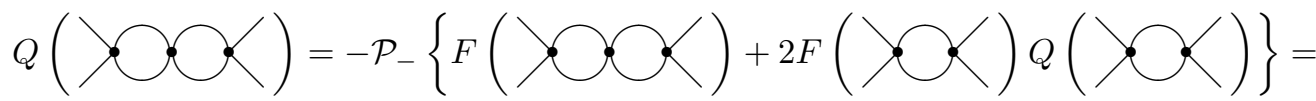

$$
\begin{aligned}
=- & {\left[\left(\frac{i}{32 \pi^{2}}\right)^{2}\left\{\ln ^{2}\left(\frac{M^{2}}{\mu^{2}}\right)-2 \ln \left(\frac{M^{2}}{\mu^{2}}\right) I_{\text {fin }}^{(1, s)}\left(p^{2}\right)\right\}+\right.} \\
& \left.+2 \times \frac{i}{32 \pi^{2}}\left\{-\ln \left(\frac{M^{2}}{\mu^{2}}\right)+I_{\text {fin }}^{(1, t)}\left(p^{2}\right)\right\}\left\{\frac{i}{32 \pi^{2}} \ln \left(\frac{M^{2}}{\mu^{2}}\right)\right\}\right]=
\end{aligned}
$$$$
=\left(\frac{i}{32 \pi^{2}}\right)^{2} \ln ^{2}\left(\frac{M^{2}}{\mu^{2}}\right) \text {, }
$$

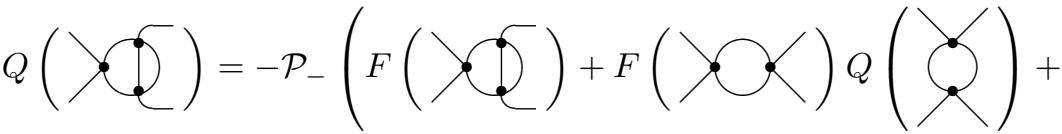

$$
\begin{aligned}
& +F(\bigotimes) Q(\oiiint))= \\
& =-\left(\frac{i}{32 \pi^{2}}\right)^{2} \mathcal{P}_{-}\left[\ln ^{2}\left(\frac{M^{2}}{\mu^{2}}\right)-2 \ln \left(\frac{M^{2}}{\mu^{2}}\right) I_{\text {fin }}^{(1, s)}\left(p^{2}\right)+\right. \\
& \left.+I_{\text {fin }}^{(2, t \cdot(s+u))}\left(p^{2}\right)-2\left\{-\ln \left(\frac{M^{2}}{\mu^{2}}\right)+I_{\text {fin }}^{(1, t)}\left(p^{2}\right)\right\} \ln \left(\frac{M^{2}}{\mu^{2}}\right)\right]= \\
& =-3\left(\frac{i}{32 \pi^{2}}\right)^{2} \ln ^{2}\left(\frac{M^{2}}{\mu^{2}}\right) \text {. }
\end{aligned}
$$


Собирая все $Q$ в одну формулу для $\widetilde{T}$, мы получим:

$$
\begin{aligned}
-i \widetilde{T} & =-i T+3 \frac{i}{32 \pi^{2}} \ln \left(\frac{M^{2}}{\mu^{2}}\right)(-i T)^{2}-3\left(\frac{i}{32 \pi^{2}}\right)^{2} \ln ^{2}\left(\frac{M^{2}}{\mu^{2}}\right)(-i T)^{3}, \\
\widetilde{T} & =T+\frac{3}{32 \pi^{2}} \ln \left(\frac{M^{2}}{\mu^{2}}\right) T^{2}-\frac{3}{\left(32 \pi^{2}\right)^{2}} \ln ^{2}\left(\frac{M^{2}}{\mu^{2}}\right) T^{3} .
\end{aligned}
$$

Такое $\widetilde{T}$ действительно делает $F(T) \mathcal{P}$-положительной, и опять-таки ответ для $F(\widetilde{T})$ может быть напрямую получен из формулы лесов второго уровня (5):

$$
\begin{aligned}
& F_{\mathrm{R}}(T)=F(\widetilde{T}), \\
& F_{\mathrm{R}}(\chi)=F(\chi)=1 \text {, }
\end{aligned}
$$

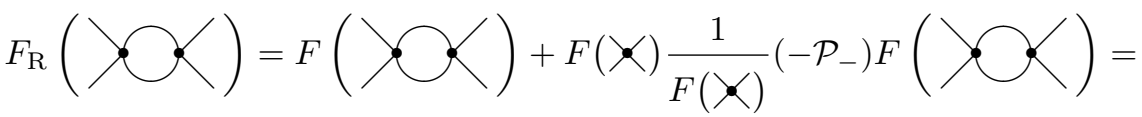

$$
\begin{aligned}
& =\mathcal{P}_{+} F(\bigcirc)=\frac{i}{32 \pi^{2}} \int_{0}^{1} d x \ln \frac{m^{2}-x(1-x) p^{2}}{\mu^{2}} .
\end{aligned}
$$

На уровне двухпетлевых диаграмм мы должны просуммировать по следующим лесам для
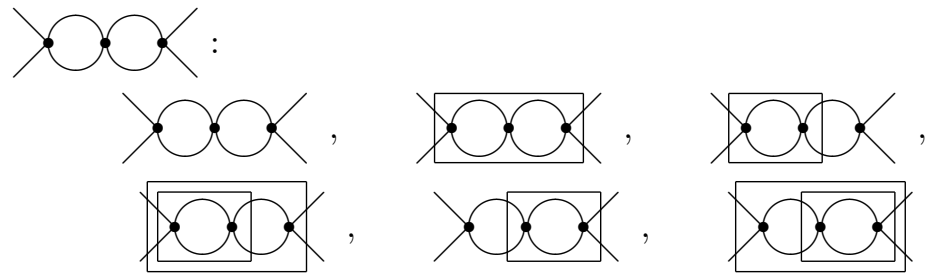

и по следующим лесам для
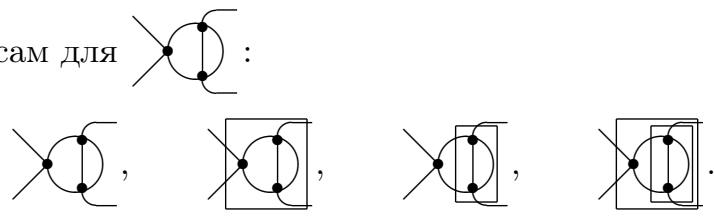

В соответствии с правилами, описанными в п. 1.3, лесу ражение

$$
\left(-\mathcal{P}_{-}\right) F(欠)
$$

(мы опускаем $F(\chi)=1$ ), в то время как

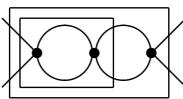
отвечает

$$
\left(-\mathcal{P}_{-}\right)\left\{F(囚)\left(-\mathcal{P}_{-}\right) F(\bigcirc)\right\}
$$

Изобразим действие $\mathcal{P}$ - на диаграмму, обводя ее овалом, и обозначим $\odot$ вершину, которая содержала стянутый в точку граф. Тогда мы можем символически написать выражение для $R(\Gamma) \equiv F_{\mathrm{R}}(\Gamma)$ как

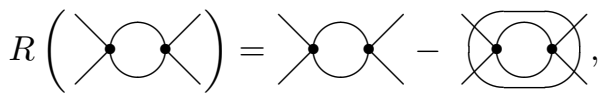

5 Теоретическая и математическая физика, т. 154, № 2, 2008 г. 


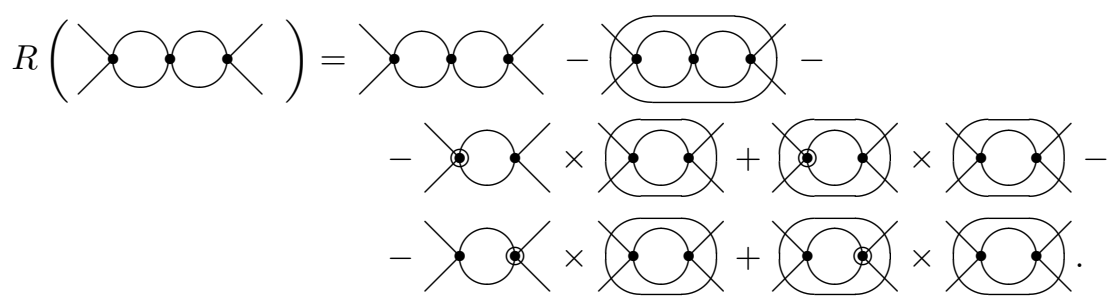

В $R(\Gamma) \equiv F_{\mathrm{R}}(\Gamma)$ легко узнать $R$-операцию Боголюбова [1], [2] вместе с ее графическим представлением в формулах (58) и (59).

\section{II.2. ПЕРЕНОРМИРУЕМАЯ И НЕПЕРЕНОРМИРУЕМАЯ МОДЕЛИ КВАНТОВОЙ ТЕОРИИ ПОЛЯ $\phi^{4}$ В $d=4$ И $\mathbf{B} d \geqslant 5$}

В данном разделе мы кратко затронем различие между перенормируемыми и неперенормируемыми моделями квантовой теории поля. Поскольку формулу лесов необходимо применять порядок за порядком по константе связи, следует рассматривать все графы в данном порядке. Возвращаясь к уравнению (51) для теории $\phi^{4}$, мы видим, что в третьем порядке имеются дополнительные диаграммы, к примеру

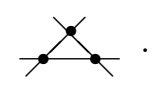

Мы опустили эти диаграммы в (51), так как при $d=4$ выражение для них не содержит расходящихся слагаемых:

$$
\mathcal{P}_{-}\{F(,)\}=0 .
$$

Однако это утверждение зависит от размерности пространства-времени $d$, и эта диаграмма расходится как $M^{d-6}$ при $d \geqslant 6$. При $d=5$ эта диаграмма конечна, однако расходится другая диаграмма

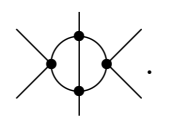

Таким образом, для того чтобы выполнялся вершинный критерий и мы могли самосогласованно перенормировать нашу теорию, необходимо дополнительно включить в $F(T)$ шестивалентную вершину $T_{6}$, добавив ее к элементарной четырехвалентной вершине $T_{4}$ :

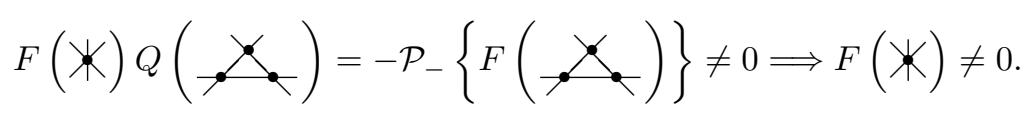

После этого нам следует учесть вклад от всех диаграмм, появившихся в результате добавления новой вершины, в данном (третьем) порядке. Дополнительные диаграммы, требующие учета, например, таковы:
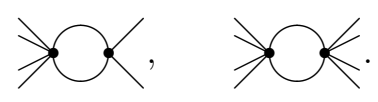
И та, и другая диаграммы расходятся, так как в них содержится петля с двумя пропагаторами, и они дают вклад в $Q(\Gamma)$. Первая диаграмма дает поправку к $T_{6}$, однако вторая диаграмма генерирует $T_{8}$ - элементарную восьмивалентную вершину:

$$
F(*) Q(\nVdash<)=-\mathcal{P}_{-}\{F(\nVdash<)\} \neq 0 \Longrightarrow F(\nVdash) \neq 0 \text {; }
$$

вершина $T_{8}$, в свою очередь, генерирует $T_{10}$ и т.д. Мы видим, что хотя при $d \geqslant 5$ перенормировка $F(T)$ и возможна, но для того чтобы ее провести самосогласованно, требуется добавить бесконечное количество новых вершин (и констант связи) даже в третьем порядке по T. Такие теории называются неперенормируемыми.

Ситуация в размерности $d=4$ отличается от той, которая имеет место в более высоких размерностях. Поскольку выполняется (61), диаграмма (60) не дает вклада в перенормировку $F(T)$ из уравнения (51) и не генерирует новых вершин. Это свойство не связано с тем, что мы ограничились рассмотрением третьего порядка по T. Можно показать [1]-[3], что все расходимости в диаграммах с более чем четырьмя внешними линиями возникают из-за расходящихся подграфов с четырьмя концами. $\mathrm{K}$ примеру, хотя в размерности $d=4$

$$
\mathcal{P}_{-}\left\{F\left(\bigcirc_{-}\right)\right\} \neq 0
$$

эта расходимость не требует введения шестивалентной вершины взаимодействия, так как она в точности сокращается в формуле лесов:

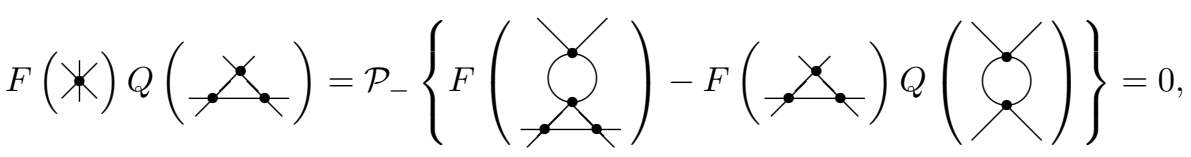

и мы можем согласованно положить $F(\chi)=0$. На языке перенормированной теории возмущений это означает, что все расходимости могут быть поглощены единственным контрчленом для четырехвалентной вершины: $Q(\Gamma)$, которое делает $F(T)$ или какой-либо другой коррелятор в нашей теории конечным, принимает ненулевые значения только на диаграммах с четырьмя внешними линиями. Теории, удовлетворяющие этому условию, называются перенормируемыми. 


\section{ПРИЛОЖКНИЕ}

1. Доказательство формулы лесов. После большого числа примеров, которые мы рассмотрели в данной работе, доказательство формулы лесов становится довольно простым упражнением. Напомним, что для данной функции $F(T)$ и линейного проектора $\mathcal{P}_{-}$формула лесов дает решение уравнения $(1)$, позволяя найти сдвиг переменной $T \rightarrow \widetilde{T}=T+Q(T)$. Другими словами, формула лесов позволяет изменить вид функции $F$ требуемым образом путем сдвига аргумента функции, не изменяя при этом вид самой функции. Это важно для физических применений, поскольку таким образом можно избавится от проблем, возникающих в статсумме (т.е. от расходимостей), не нарушая ее свойств (скажем, оставляя статсумму в узком классе интегрируемых $\tau$-функций).

Записав представление

$$
F(T+Q(T))=\sum_{\Gamma} \widehat{F}(\Gamma) Z(\Gamma \mid T+Q(T))=\sum_{\Gamma} \widehat{F}_{\mathrm{R}}(\Gamma) Z(\Gamma \mid T),
$$

мы видим, что, поскольку функции $Z(\Gamma \mid T)$ образуют базис в пространстве всех функций переменной $T$, уравнение (1) будет выполняться тогда и только тогда, когда $\mathcal{P}_{-} F_{\mathrm{R}}(\Gamma)=0$ для всех $\Gamma$ (напоминаем, что проектор $\mathcal{P}_{-}$не действует на $Z(\Gamma \mid T))$. Для того чтобы найти коэффициенты $\widehat{F}_{\mathrm{R}}(\Gamma)$, мы подставляем $Q(T)=$ $\sum_{\gamma} \widehat{Q}(\gamma) Z(\gamma \mid T)$ и сравниваем слагаемые с обеих сторон равенства

$$
\sum_{\Gamma} \widehat{F}(\Gamma) Z\left(\Gamma \mid T+\sum_{\gamma} \widehat{Q}(\gamma) Z(\gamma \mid T)\right)=\sum_{\Gamma} \widehat{F}_{\mathrm{R}}(\Gamma) Z(\Gamma \mid T) .
$$

Поскольку

$$
\begin{aligned}
Z\left(\Gamma \mid T+\sum_{\gamma} \widehat{Q}(\gamma) Z(\gamma \mid T)\right)=Z(\gamma \mid T)+\sum_{\Upsilon, \gamma: \Upsilon / \gamma=\Gamma} \widehat{Q}(\gamma) Z(\Upsilon \mid T)+ \\
\quad+\sum_{\Upsilon, \gamma_{1}, \gamma_{2}: \Upsilon /\left(\gamma_{1} \gamma_{2}\right)=\Gamma} \widehat{Q}\left(\gamma_{1}\right) \widehat{Q}\left(\gamma_{2}\right) Z(\Upsilon \mid T)+\cdots,
\end{aligned}
$$

мы получаем, что

$$
\begin{gathered}
\widehat{F}_{\mathrm{R}}(\Gamma)=\widehat{F}(\Gamma)+\sum_{\substack{\left\{\gamma_{1} \cup \cdots \cup \gamma_{k}\right\} \\
\mathcal{P}_{-} \widehat{F}_{\mathrm{R}}(\Gamma)=0,}} \widehat{F}\left(\Gamma /\left(\gamma_{1} \ldots \gamma_{k}\right)\right) \widehat{Q}\left(\gamma_{1}\right) \ldots \widehat{Q}\left(\gamma_{k}\right), \\
\mathcal{P}_{-}\left\{\widehat{F}(\Gamma)+\widehat{F}(\Gamma / \Gamma) \widehat{Q}(\Gamma)+\sum_{\left\{\gamma_{1} \cup \cdots \cup \gamma_{k}\right\}}^{\prime} \widehat{F}\left(\Gamma /\left(\gamma_{1} \ldots \gamma_{k}\right)\right) \widehat{Q}\left(\gamma_{1}\right) \ldots \widehat{Q}\left(\gamma_{k}\right)\right\}=0,
\end{gathered}
$$

где штрих над знаком суммы означает что мы исключаем из суммирования слагаемое с $\gamma=\Gamma$. Теперь, считая, что $\mathcal{P}_{+} \widehat{F}(\Gamma / \Gamma) \widehat{Q}(\Gamma)=0$, мы получаем формулу лесов (4):

$$
\widehat{F}(\Gamma / \Gamma) \widehat{Q}(\Gamma)=-\mathcal{P}_{-}\left\{\widehat{F}(\Gamma)+\sum_{\left\{\gamma_{1} \cup \ldots \cup \gamma_{k}\right\}} \widehat{F}\left(\Gamma /\left(\gamma_{1} \ldots \gamma_{k}\right)\right) \widehat{Q}\left(\gamma_{1}\right) \ldots \widehat{Q}\left(\gamma_{k}\right)\right\}
$$


что и требовалось доказать.

Формула лесов второго уровня (5) может быть получена решением рекурсии для $\widehat{Q}(\Gamma)$ и подстановкой результата в (П.1).

\section{2. Кинетические слагаемые, одночастично приводимые диаграммы и} другие особенности. Здесь мы кратко прокомментируем особенности, которые не были затронуты в несколько упрощенном рассмотрении в разделе II.1.

В первую очередь надо объяснить ситуацию с одночастично приводимыми (ОЧП) диаграммами. Они были исключены из рассмотрения в разделе II.1, поскольку не дают вклада в контрчлены $Q$. Это можно легко доказать по индукции. Действительно, рассмотрим ОЧП-граф

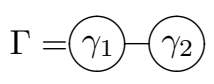

(внешние концы на рисунке не показаны). Его фейнмановская амплитуда дается произведением пропагатора и двух амплитуд для одночастично неприводимых $(\mathrm{OЧН})$ поддиаграмм $\gamma_{1}, \gamma_{2}$. Предположив, что $Q$ для всех ОЧП-диаграмм с меньшим числом вершин обращается в нуль, мы получаем, что множество прямоугольных поддиаграмм диаграммы $\Gamma$, которые в принципе могут дать вклад в $Q(\Gamma)$, состоит из объединения соответствующих множеств для ОЧН-поддиаграмм и прямоугольников, полностью содержащих в себе $\gamma_{1}$ или $\gamma_{2}$. Таким образом, мы получаем

$$
Q(\Gamma)=\mathcal{P}_{-}\left\{\left(F\left(\gamma_{1}\right)-Q\left(\gamma_{1}\right)\right)\left(F\left(\gamma_{2}\right)-Q\left(\gamma_{2}\right)\right)\right\} \times\{-\}=0,
$$

поскольку

$$
\mathcal{P}_{-}\left\{F\left(\gamma_{1}\right)-Q\left(\gamma_{1}\right)\right\}=\mathcal{P}_{-}\left\{F\left(\gamma_{2}\right)-Q\left(\gamma_{2}\right)\right\}=0
$$

Этот вывод, очевидно, основывается на том, что все расходимости в КТП возникают из-за петель, и на том, что ОЧП-диаграмма равна произведению своих ОЧН-компонент.

Кроме этого, требует пояснения перенормировка массового и кинетического слагаемых. Для рассмотрения этой проблемы нам необходимо ввести две дополнительные константы связи в наше рассмотрение в разделе II.1 и, соответственно, две дополнительные элементарные вершины $-\bullet,-<$, первая из которых отвечает массовому слагаемому, а вторая - кинетическому. Лагранжиан и элементарные вершины взаимодействия теперь принимают следующий вид:

$$
\begin{gathered}
L(\phi)=\frac{\kappa}{2}\left(\partial_{\mu} \phi\right)^{2}-\frac{1}{2} m^{2} \phi^{2}-\frac{T}{4 !} \phi^{4} ; \\
F(\bullet)=1, \quad F(\star)=p^{2}, \quad F(\ngtr)=1 ; \\
Z(\bullet \mid T)=m^{2}, \quad Z(\star \mid T)=\kappa, \quad Z(\Varangle \mid T)=-i T .
\end{gathered}
$$

Обычно предполагают, что физическое значение $\tilde{\kappa}=1$, но голое значение этой константы может отличаться от единицы. 
Теперь мы можем записать сумму всех корреляторов, которые в принципе могут дать вклад в $Q$, т.е. корреляторов, которые потенциально расходятся:

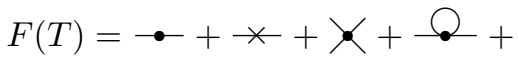

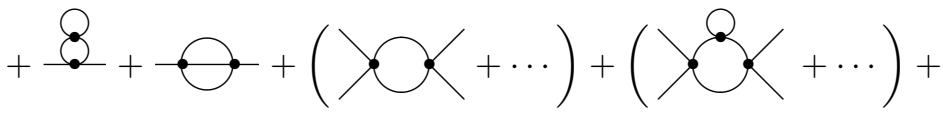

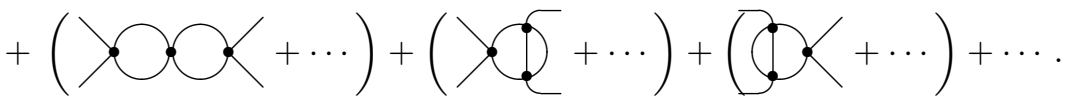

Так как имеются две различные элементарные вершины, отвечающие двум различным константам связи, нам необходимо ввести дополнительную внешнюю структуру, чтобы разделить вклады в $Q$ на две части: $Q$ для диаграмм с двумя внешними линиями приобретает дополнительный индекс, равный 0 или 1 . Мы имеем два проектора $\mathcal{P}_{-}^{(0)}$ и $\mathcal{P}_{-}^{(1)}$ для диаграмм собственной энергии, $\mathcal{P}_{-}^{(0)}$ выбирает сингулярные слагаемые, пропорциональные $p^{0}$, в то время как $\mathcal{P}_{-}^{(1)}$ оставляет слагаемые, пропорциональные $p^{2}$. Соответственно

$$
Q^{(i)}(\Gamma)=-\mathcal{P}_{-}^{(i)}\{F(\Gamma)+\cdots\}
$$

Теперь мы можем выполнять перенормировку, "как обычно":

$$
\begin{gathered}
\tilde{m}^{2}=m^{2}+\sum_{\substack{\text { self-energy } \\
\text { graphs }}} Q^{(0)}(\Gamma), \\
\tilde{\kappa}=\kappa+\sum_{\substack{\text { self-energy } \\
\text { graphs }}} Q^{(1)}(\Gamma), \\
-i \widetilde{T}=-i T+\sum_{\substack{\text { graphs with } 4 \\
\text { external legs }}} Q(\Gamma) .
\end{gathered}
$$

Более подробное рассмотрение перенормировки массовых слагаемых можно найти в работах [6], [8] для теории $\phi^{3}$, в работе [5] для теории $\phi^{4}$ и в работах [8], [9] для квантовой электродинамики.

Благодарности. Наша работа частично поддержана Фондом "Династия" (М.Н. Сербин), РФФИ (гранты № 07-02-00878, 07-02-00645, объединенный грант № 06-01-92059-CE), NWO (проект № 047.011.2004.026), INTAS (грант № 05-10000087865), Программой поддержки ведущих научных школ (грант № НШ-8004.2006.2), а также проектом ANR-05-BLAN-0029-01 (А. Ю. Морозов).

\section{Список литературы}

[1] N. N. Bogoliubow, O.S. Parasiuk, Acta Math., 97 (1957), 227-266; Н. Н. Боголюбов, Д. В. Ширков,, Введение в теорию квантованных полей, Гостехиздат, М., 1957; Б. М. Степанов, О. И. Завьялов, ЯФ, 1 (1965), 922; К. Нерp, Comm. Math. Phys., 2 (1966), 301-326; M. Zimmerman, Comm. Math. Phys., 15 (1969), 208-234. 
[2] О. И. Завьялов, Перенормированные диаграммы Феймана, Наука, М., 1979.

[3] М. Пескин, Д. Шредер, Введение в квантовую теорию поля, РХД, М.-Ижевск, 2001.

[4] Дж. Коллинз, Перенормировка, Мир, М., 1988.

[5] А.Н. Васильев, Квантовополевая ренормгруппа в теории критического поведения и стохастической динамике, Изд-во ПИЯФ, СПб., 1998.

[6] A. Connes, D. Kreimer, Comm. Math. Phys., 210 (2000), 249-273; arXiv: hep-th/9912092; Comm. Math. Phys., 216 (2001), 215-241; arXiv: hep-th/0003188.

[7] A. Gerasimov, A. Morozov, K. Selivanov, Internat. J. Modern Phys. A, 16 (2001), 1531-1558; arXiv: hep-th/0005053.

[8] D. Kreimer, R. Delbourgo, Phys. Rev. D (3), 60 (1999), 105025; arXiv: hep-th/9903249; K. Ebrahimi-Fard, D. Kreimer, J. Phys. A, 38 (2005), R385-R407; arXiv: hep-th/0510202; D. Kreimer, "Structures in Feynman graphs: Hopf algebras and symmetries", Graphs and Patterns in Mathematics and Theoretical Physics, Proc. Sympos. Pure Math., 73, AMS, Providence, RI, 2005, 43-78; arXiv: hep-th/0202110; Ann. Phys., 321 (2006), 2757-2781; arXiv: hep-th/0509135.

[9] W. D. van Suijlekom, Lett. Math. Phys., 77 (2006), 265-281; arXiv: hep-th/0602126.

[10] R. Wulkenhaar, "Hopf algebras in renormalization and NC geometry", Noncommutative geometry and the standard model of elementary particle physics (Hesselberg, Germany, 1999), Lecture Notes in Phys., 596, Springer, Berlin, 2002, 313-324; arXiv: hep-th/9912221.

[11] Д. В. Малышев, ТМФ, 143:1 (2005), 22-32; arXiv: hep-th/0408230; D. V. Malyshev, Non $R G$ logarithms via $R G$ equations, arXiv: hep-th/0402074; Phys. Lett. B, 578 (2004), 231-234; arXiv: hep-th/0307301.

[12] D. I. Kazakov, G.S. Vartanov, Renormalizable expansion for nonrenormalizable theories: I. Scalar higher dimensional theories, arXiv: hep-th/0607177.

[13] D. I. Kazakov, G. S. Vartanov, Renormalizable expansion for nonrenormalizable theories: II. Gauge higher dimensional theories, arXiv: hep-th/0702004.

[14] И. В. Волович, Д.В. Прохоренко, Тр. МИАН, 245 (2004), 288-295; arXiv: hep-th/0611178.

[15] B. Delamotte, Amer. J. Phys., 72:2 (2004), 170-184; arXiv: hep-th/0212049.

[16] V. Dolotin, A. Morozov, Introduction to Non-Linear Algebra, World Sci., Singapore, 2007; arXiv: hep-th/0609022. 